Pacific

Journal of

Mathematics

A TOPOLOGICAL APPROACH TO INVERSE AND REGULAR SEMIGROUPS

BenJAMin Steinberg

Volume $208 \quad$ No. 2

February 2003 


\title{
A TOPOLOGICAL APPROACH TO INVERSE AND REGULAR SEMIGROUPS
}

\author{
Benjamin SteinberG
}

\begin{abstract}
Work of Ehresmann and Schein shows that an inverse semigroup can be viewed as a groupoid with an order structure; this approach was generalized by Nambooripad to apply to arbitrary regular semigroups. This paper introduces the notion of an ordered 2-complex and shows how to represent any ordered groupoid as the fundamental groupoid of an ordered 2-complex. This approach then allows us to construct a standard 2-complex for an inverse semigroup presentation.

Our primary applications are to calculating the maximal subgroups of an inverse semigroup which, under our topological approach, turn out to be the fundamental groups of the various connected components of the standard 2-complex. Our main results generalize results of Haatja, Margolis, and Meakin giving a graph of groups decomposition for the maximal subgroups of certain regular semigroup amalgams. We also generalize a theorem of Hall by showing the strong embeddability of certain regular semigroup amalgams as well as structural results of Nambooripad and Pastijn on such amalgams.
\end{abstract}

\section{Introduction.}

In the fifties, there were two attempts to axiomatize the underlying structure of pseudogroups of diffeomorphisms of manifolds. One approach, by Wagner (and independently by Preston [16]), was via inverse semigroups; the other, by Ehresmann, was via ordered groupoids, namely the so-called inductive groupoids popularized amongst semigroup theorists by Schein [18]. It is fascinating that some results are proved more easily via the inverse semigroup approach, while others are more naturally proved from the point of view of ordered groupoids. In his seminal paper [11], Nambooripad extended this equivalence to an equivalence between regular semigroups and, what we shall call in this paper, $r$-inductive groupoids. Recent work [15] emphasizes the importance of inverse semigroups in the theory of $C^{*}$-algebras.

In this paper, building on the idea that any groupoid can be realized as the fundamental groupoid of a 2-complex, we introduce the notion of an ordered 2-complex and show that any ordered groupoid can be realized as 
the fundamental ordered groupoid of an ordered 2-complex. In the process, we introduce the notion of a presentation of an ordered groupoid. While an inverse semigroup presentation is different from an ordered groupoid presentation, we show how to construct an ordered groupoid presentation from any inverse semigroup presentation by constructing what we call the standard ordered 2-complex of an inverse semigroup presentation (generalizing the usual notion from group presentations). This complex is closely related to the Munn and right letter mapping representations of the inverse semigroup.

The maximal subgroups of the inverse (regular) semigroup will turn out to be the fundamental groups of the connected components of an ordered 2 -complex representing the semigroup. This will allow us to obtain a quick topological proof that if a maximal subgroup of a finitely presented inverse semigroup acts on the left of its $\mathcal{R}$-class with finite quotient, then it is finitely presented.

We also introduce the Schützenberger complex of an inverse semigroup presentation. This complex is a $\pi_{1}$-trivial covering space of the standard ordered 2-complex of the presentation and plays a role in the theory similar to that played by the Cayley complex (which it generalizes) in group theory. The 1-skeleton of the Schützenberger complex is the union of all the Schützenberger graphs of the presentation [24]. This complex and the standard ordered 2-complex, both of which are built from semigroup theoretic tools, provide a link between the semigroup and ordered groupoid approaches to the subject.

Our main result is a structure theorem for maximal subgroups of certain amalgams. In [3], it is proved that a maximal subgroup of a full amalgam of regular semigroups has a certain graph of groups decomposition. In this paper, we are able to weaken the restrictions and, at the same time, obtain a much simpler proof. The proof of $[\mathbf{3}]$ relies on Bass-Serre theory whence they have to construct an action of the maximal subgroup on a tree with appropriate stabilizers; they use normal forms for the amalgamated product in order to do this. Our approach is to show that the amalgamated product can be represented by a topological space which is a segment of disconnected 2-complexes; the structure of the maximal subgroups then follows from an obvious decomposition of such a graph of 2-complexes into a graph of connected 2-complexes. In the process, we extend a result of Hall [4] on the strong embeddability of regular semigroup amalgams as well as structural results of Nambooripad and Pastijn [12] on such. We obtain further results for certain inverse semigroup amalgams, overlapping at times with results of Bennet [1]. We also obtain results for certain amalgams in the category of inverse semigroups and prehomomorphisms. For some related results on inverse semigroup amalgams, see $[\mathbf{2 2}]$. 
Future work, will show that the various structure theorems for idempotent pure morphisms of inverse semigroups correspond to facts about when a morphism of ordered 2-complexes can be extended nicely to a covering.

\section{Preliminaries.}

If $A$ is a set, we let $\widetilde{A}=A \cup A^{-1}$. Then $\widetilde{A}^{+}$will denote the free semigroup and $\widetilde{A}^{*}$ the free monoid with involution on $A$.

If $S$ is a semigroup, an element $e \in S$ is an idempotent if $e^{2}=e$. The set of idempotents of $S$ will be denoted $\mathcal{E}(S)$. An element $s \in S$ is called regular if $s=s t s$ for some $t \in S$. A semigroup in which each element is regular is called a regular semigroup. If $s$ and $t$ are such that $s t s=s$ and $t s t=t$, then $s$ and $t$ are said to be inverses of each other. One can show that an element is regular if and only if it has an inverse [6]. A semigroup $S$ is called an inverse semigroup if each element $s \in S$ has a unique inverse (denoted $s^{-1}$ ) or, equivalently, if it is regular and has commuting idempotents [7]. The notation $S^{1}$ is used for the monoid obtained by adding an identity to $S$. We refer the reader to [7] for more on inverse semigroups. In this paper, we shall primarily focus on inverse semigroups, only occasionally speaking of more general regular semigroups.

We briefly recall Green's relations [6]. If $S$ is a semigroup, then we write $s \leq_{\mathcal{R}} t$ if $s S^{1} \subseteq t S^{1}$; this is a preorder and we use $\mathcal{R}$ for the associated equivalence relation. The preorder $\leq_{\mathcal{L}}$ and equivalence relation $\mathcal{L}$ are defined dually. One sets $\mathcal{H}=\mathcal{R} \cap \mathcal{L}$ and $\mathcal{D}=\mathcal{R} \circ \mathcal{L} ; \mathcal{D}$ is the smallest equivalence relation containing $\mathcal{R}$ and $\mathcal{L}$. If $e \in \mathcal{E}(S)$, then the $\mathcal{H}$-class of $e$ is the maximal subgroup with identity $e[6]$. For an inverse semigroup, $s \mathcal{R} t$ if and only if $s s^{-1}=t t^{-1}[7]$ and dually for $\mathcal{L}$.

If $I$ is an inverse semigroup, there is a natural partial order on $I$ defined by $s \leq t$ if $s=s s^{-1} t$. This order is well-known to be compatible with the operations of multiplication and taking inverses [7].

Inverse semigroups can be viewed as a variety of unary semigroups and as such there is a free inverse semigroup on any set as well as a notion of inverse semigroup presentations; see [24] for some graph-theoretical techniques for working with inverse semigroup presentation.

A morphism $\varphi$ of partially ordered sets is called an order embedding if $\varphi(x) \leq \varphi(y)$ if and only if $x \leq y$. A surjective order embedding is called an order isomorphism.

\section{Ordered graphs.}

Following the Serre convention [19], we define a graph $X$ to consist of: A set $V(X)$ of vertices; a set $E(X)$ of edges; an involution $e \mapsto e^{-1}$ on $E(X)$; and a function $\mathbf{d}: E(X) \rightarrow V(X)$ which selects the initial vertex of an edge (the terminology $\mathbf{d}$ is chosen to suggest the word domain). We define 
$\mathbf{r}: E(X) \rightarrow V(X)$ by $\mathbf{r}(e)=\mathbf{d}\left(e^{-1}\right)$ (and $\mathbf{r}(e)$ is called the terminal vertex of $e$ ). One defines, for $v \in V(X), \operatorname{Star}(v)=\mathbf{d}^{-1}(v)$. In general, we shall require $e^{-1} \neq e$, the exception being when we view groupoids as graphs in which case local group elements of order 2 will have this property. Our reason for choosing this unorthodox notation for graphs is because many of our graphs will, in fact, be groupoids, where the terms domain and range have obvious meanings.

Graph morphisms are defined in the obvious way. If $\varphi: X \rightarrow Y$ is a graph morphism and, for each $v \in V(X), \varphi: \operatorname{Star}(v) \rightarrow \operatorname{Star}(\varphi(v))$ is, respectively, injective, surjective, bijective, then $\varphi$ is called, respectively, an immersion, a fibration, a covering.

An ordered graph is a graph $X$ such that: $V(X)$ and $E(X)$ are partially ordered sets; the involution and $\mathbf{d}$ preserve order (whence $\mathbf{r}$ preserves order); and if $e \in E(X), v \in V(X)$ are such that $v \leq \mathbf{d}(e)$, then there is a unique edge of $X$, denoted $(v \mid e)$, with $\mathbf{d}(v \mid e)=v$ and $(v \mid e) \leq e$, called the restriction of e to $v$. Note that this third condition implies that the edges of $\operatorname{Star}(v)$ are incomparable for any vertex $v$. Observe that if $v \leq \mathbf{d}(e)$, then $(v \mid e)^{-1}=\left(\mathbf{r}(v \mid e) \mid e^{-1}\right)$. Morphisms of ordered graphs are graph morphisms which preserve the partial order.

If $v_{1}, v_{2} \in V(X)$ and $v_{1} \leq v_{2}$, we can define a restriction map res $_{v_{2}}^{v_{1}}$ : $\operatorname{Star}\left(v_{2}\right) \rightarrow \operatorname{Star}\left(v_{1}\right)$ by $\operatorname{res}_{v_{2}}^{v_{1}}(e)=\left(v_{1} \mid e\right)$. The above, together with straightforward reasoning, shows that these restriction maps satisfy the following three properties:

(1) $v_{1} \leq v_{2} \leq v_{3} \Longrightarrow \operatorname{res}_{v_{2}}^{v_{1}} \operatorname{res}_{v_{3}}^{v_{2}}=\operatorname{res}_{v_{3}}^{v_{1}}$;

(2) $\mathbf{r}\left(\operatorname{res}_{v_{2}}^{v_{1}}(e)\right) \leq \mathbf{r}(e)$;

(3) $\operatorname{res}_{v_{2}}^{v_{1}}(e)^{-1}=\operatorname{res}_{\mathbf{r}(e)} \mathbf{r}\left(\operatorname{res}_{v_{2}}^{v_{1}}(e)\right)\left(e^{-1}\right)$.

Any morphism of ordered graphs automatically preserves these restrictions maps.

Conversely, given a graph $X$ such that $V(X)$ is partially ordered and given $\operatorname{maps}_{v_{2}}^{v_{1}}: \operatorname{Star}\left(v_{2}\right) \rightarrow \operatorname{Star}\left(v_{1}\right)$, whenever $v_{1} \leq v_{2}$, satisfying the above three properties, we can turn $X$ into an ordered graph by defining, for $e_{1}, e_{2} \in E(X), e_{1} \leq e_{2}$ if $\mathbf{d}\left(e_{1}\right) \leq \mathbf{d}\left(e_{2}\right)$ and $e_{1}=\operatorname{res}_{\mathbf{d}\left(e_{2}\right)}^{\mathbf{d}\left(e_{1}\right)}\left(e_{2}\right)$. We shall use freely throughout these two equivalent formulations of the definition of an ordered graph.

An ordered subgraph of an ordered graph is a subgraph which is an ordered graph with the induced ordering (that is, a subgraph closed under restrictions).

\section{Ordered groupoids.}

If $G$ is an ordered graph, then two edges $e_{1}, e_{2}$ are said to be composable if $\mathbf{r}\left(e_{1}\right)=\mathbf{d}\left(e_{2}\right)$. An ordered groupoid is an ordered graph with an associative 
multiplication on composable edges satisfying, whenever the compositions make sense:

(1) $x x^{-1} y=y$;

(2) $x_{1} \leq y_{1}, x_{2} \leq y_{2} \Longrightarrow x_{1} x_{2} \leq y_{1} y_{2}$.

The first condition implies that at each vertex $v$ there is a unique identity which will be denoted $1_{v}$. It is easy to show that the map $v \mapsto 1_{v}$ is an order isomorphism.

In terms of the restriction maps, the second condition becomes: If $x_{1} x_{2}$ is defined and $v \leq \mathbf{d}\left(x_{1}\right)$, then

$$
\left(v \mid x_{1} x_{2}\right)=\left(v \mid x_{1}\right)\left(\mathbf{r}\left(v \mid x_{1}\right) \mid x_{2}\right) .
$$

Remember that, for groupoids, we do allow $e=e^{-1}$.

An ordered subgroupoid of an ordered groupoid is a subgroupoid which is an ordered subgraph.

An inductive groupoid is an ordered groupoid $G$ in which $V(G)$ is a semilattice; we shall call $G r$-inductive if $V(G)$ is a biordered set [11]. These definitions make perfect sense for ordered graphs and we shall extend their domains to that realm. The notions of ordered and inductive groupoids are due to Ehresmann [2]; see also Schein [18].

Any inverse semigroup $I$ can be realized as an inductive groupoid as follows: $V(I)=\mathcal{E}(I) ; E(I)=I$; the order is the natural partial order; the inverse is the inverse; $\mathbf{d}(s)=s s^{-1}$; and the product is the usual multiplication. Conversely, given an inductive groupoid $I$, we can define an inverse semigroup whose elements are $E(I)$. If $s, t \in E(I)$, we define

$$
s t=\left(\mathbf{r}(s) \wedge \mathbf{d}(t) \mid s^{-1}\right)^{-1}(\mathbf{r}(s) \wedge \mathbf{d}(t) \mid t) .
$$

These two constructions are inverse to each other; see [7] for more details. We observe that the $\mathcal{R}$-class of an idempotent $e \in \mathcal{E}(I)$ is $\operatorname{Star}(e)$, the $\mathcal{H}$-class of $e$ is the local group at $e$ (see below for the definition), and the $\mathcal{D}$-class of $e$ is the connected component of the groupoid containing $e$. Inverse semigroup homomorphisms correspond to ordered groupoid morphisms which preserve the inductive structure.

There is a similar, but more complicated, correspondence between regular semigroups and $r$-inductive groupoids [11].

A groupoid in the usual sense is an ordered groupoid under the equality ordering. In particular, any group is an inductive groupoid.

If $v \in V(G)$ is a vertex of an ordered groupoid $G$, then

$$
G_{v}=\{g \in E(G) \mid \mathbf{d}(g)=v=\mathbf{r}(g)\}
$$

is a group called the local group or maximal subgroup of $G$ at $v$.

An order ideal in a partially ordered set is a subset $I$ such that $x \leq$ $y \in I$ implies $x \in I$. If $G$ is an ordered groupoid, we use $\operatorname{Id}(G)$ to denote 
the ordered subgroupoid consisting of all vertices and identities. It can be shown [7] that $E(\operatorname{Id}(G))$ is an order ideal in $E(G)$.

\section{Paths and free ordered groupoids.}

A path in a graph is defined in the usual way; we allow an empty path at each vertex. If $p$ is a path, we shall use $\mathbf{d}(p)$ for its initial vertex, $\mathbf{r}(p)$ for its terminal vertex, and $p^{-1}$ for the reverse path. A path $p$ is said to be closed if $\mathbf{d}(p)=\mathbf{r}(p)$. If $p$ is a closed path, we say that a path $p^{\prime}$ is a cyclic conjugate of $p$ if $p=s t$ and $p^{\prime}=t s$ (that is, $p$ and $p^{\prime}$ are "the same path" starting from different vertices). A closed path is called simple if the only repetition of vertices when tracing the path occurs when one reaches the end. Connected components of a graph are defined in the usual way. Using terminology suggestive of semigroup theory, connected components will also be called $\mathcal{D}$-classes. If $v$ is a vertex, then $D_{v}$ will denote the $\mathcal{D}$-class of $v$. Note that all maximal subgroups at vertices of the same $\mathcal{D}$-class are isomorphic.

We say that a path $p$ is obtained from a path $p^{\prime}$ by an elementary homotopy if $p$ is gotten from $p^{\prime}$ by inserting or deleting a subpath of the form $e e^{-1}$. One says that paths $p$ and $p^{\prime}$ are homotopic if $p$ can be turned into $p^{\prime}$ by a finite sequence of elementary homotopies; this is an equivalence relation and equivalent elements are coterminal. It is well-known that this definition is equivalent to asking that the paths be homotopic (in the topological sense) in the geometric realization of the graph.

We now aim to prove that the fundamental groupoid of an ordered graph is an ordered groupoid in a natural way. We shall need several lemmas to achieve this. First we define restriction maps for paths which are compatible with homotopy. If $X$ is an ordered graph, $p=e_{1} e_{2} \ldots e_{n}$ is a path, and $v \leq \mathbf{d}(p)$ we define $(v \mid p)$ as follows: First set $e_{1}^{\prime}=\left(v \mid e_{1}\right)$; then, inductively, set $e_{i+1}^{\prime}=\left(\mathbf{r}\left(e_{i}^{\prime}\right) \mid e_{i+1}\right)$ for $1 \leq i \leq n-1$. It is straightforward to check that

$$
(v \mid p)=\operatorname{res}_{\mathbf{d}(p)}^{v}(p)=e_{1}^{\prime} \ldots e_{n}^{\prime}
$$

is a path starting at $v$. Observe that $\mathbf{r}(v \mid p)=\mathbf{r}\left(e_{n}^{\prime}\right) \leq \mathbf{r}\left(e_{n}\right)$. One can show by induction that if $v_{1} \leq v_{2} \leq v_{3}$, then $\operatorname{res}_{v_{2}}^{v_{1}} \operatorname{res}_{v_{3}}^{v_{2}}=\operatorname{res}_{v_{3}}^{v_{1}}$.

Lemma 5.1. Suppose $p=e_{1} \ldots e_{n}$ and $p^{\prime}=f_{1} \ldots f_{m}$ are paths in an ordered graph with $\mathbf{r}(p)=\mathbf{d}\left(p^{\prime}\right)$ and $v \leq \mathbf{d}(p)$. Then:

(1) $\left(v \mid p p^{\prime}\right)=(v \mid p)\left(\mathbf{r}(v \mid p) \mid p^{\prime}\right)$;

(2) $\operatorname{res}_{\mathbf{r}(p)}^{\mathbf{r}(v \mid p)}\left(p^{-1}\right)=(v \mid p)^{-1}$.

Proof. The inductive nature of the definition of the restriction of a path shows that to prove 1 , it suffices to show that the $(n+1)^{\text {st }}$ edge of $\left(v \mid p p^{\prime}\right)$ is $\left(\mathbf{r}(v \mid p) \mid f_{1}\right)$. Letting $e_{1}^{\prime}, \ldots, e_{n}^{\prime}$ be as above, we see that the $(n+1)^{\text {st }}$ edge is $\left(\mathbf{r}\left(e_{n}^{\prime}\right) \mid f_{1}\right)=\left(\mathbf{r}(v \mid p) \mid f_{1}\right)$ as desired. 
We note that (2) follows immediately from (1) using induction (the base case being part of the definition of an ordered graph).

We now see how elementary homotopies behave under restriction.

Lemma 5.2. Suppose that $e$ is an edge of an ordered graph and $v \leq \mathbf{d}(e)$. Then $\left(v \mid e e^{-1}\right)=(v \mid e)(v \mid e)^{-1}$.

Proof. By definition, $\left(v \mid e e^{-1}\right)=(v \mid e)\left(\mathbf{r}(v \mid e) \mid e^{-1}\right)$, but the right-hand side is $(v \mid e)(v \mid e)^{-1}$.

Corollary 5.3. If $p, p^{\prime}$ are homotopic paths in an ordered graph and $v \leq$ $\mathbf{d}(p)=\mathbf{d}\left(p^{\prime}\right)$, then $(v \mid p)$ and $\left(v \mid p^{\prime}\right)$ are homotopic.

Proof. Follows immediately from Lemmas 5.1 and 5.2.

What we have now proved is that if $X$ is an ordered graph, then the fundamental groupoid of $X$ is an ordered groupoid. That is, we define an ordered groupoid $\Pi_{1}(X)$ by taking $V\left(\Pi_{1}(X)\right)=V(X)$ and $E\left(\Pi_{1}(X)\right)$ to be the set of all homotopy classes of paths in $X$; $\mathbf{d}$ and the involution are defined in the obvious way. We can then use path composition and the path restriction maps (defined above) to turn $\Pi_{1}(X)$ into an ordered groupoid, Lemmas 5.1 and 5.2, together with Corollary 5.3, providing the necessary verifications. This groupoid is called the fundamental ordered groupoid of $X$. Its underlying groupoid is just the usual (simplicial) fundamental groupoid of $X$ (it is the subgroupoid of the topological fundamental groupoid whose vertices are vertices of $X$ and whose edges are homotopy classes of edge paths). Note that $X$ embeds in $\Pi_{1}(X)$ in a natural way and that if $X$ is an $\left(r\right.$-)inductive graph, then so is $\Pi_{1}(X)$.

We leave the following ordered analog of a standard topological fact to the reader:

Proposition 5.4. Let $X$ be an ordered graph, $G$ an ordered groupoid, and $\rho: X \rightarrow G$ a morphism of ordered graphs. Then there is a unique ordered groupoid morphism $\bar{\rho}: \Pi_{1}(X) \rightarrow G$ extending $\rho$. Furthermore, $\rho$ is a, respectively, immersion, fibration, covering if and only $\bar{\rho}$ is. In the $(r)$ inductive setting, $\rho$ preserves the $(r)$-inductive structure if and only if $\bar{\rho}$ does.

Proposition 5.4 shows that $\Pi_{1}(X)$ is the free ordered groupoid generated by $X$. To make this precise, we say that an ordered graph $X$ generates an ordered groupoid $G$ if there is a morphism $\rho: \Pi_{1}(X) \rightarrow G$ which is an order isomorphism on vertex sets and surjective on edge sets. We warn the reader that in the $(r$-)inductive setting, this concept does not correspond to a choice of semigroup generators. The kernel

$$
\operatorname{ker} \rho=\left\{p \in \Pi_{1}(X) \mid \rho(p)=1_{\mathbf{d}(p)}\right\}
$$


is then an order ideal. This leads us to the notion of an ordered groupoid presentation.

If $X$ is an ordered graph and $R$ is a collection of closed paths in $X$, then $R$ is called a set of relators if $p \in R$ and $v \leq \mathbf{d}(p)$ implies $(v \mid p) \in R$ (that is, $R$ is closed under restrictions). It then follows that the image of $R$ in $\Pi_{1}(X)$ is an order ideal. The ordered groupoid presented by generators $X$ and relators $R$, denoted $\langle X \mid R\rangle$, is then the quotient of $\Pi_{1}(X)$ obtained by identifying two paths $p, p^{\prime}$ if $p$ can be gotten from $p^{\prime}$ by a finite sequence of elementary homotopies and insertions or deletions of paths in $R \cup R^{-1}$ (or, equivalently, cyclic conjugates of such). The fact that $R$ is closed under restrictions allows one to show, similarly to the argument with elementary homotopies, that the path restrictions induce well-defined maps on the quotient groupoid. Note that every ordered groupoid $G$ has a presentation since we can choose $G$, itself, as the generating graph and all paths mapping into the kernel of the canonical surjection as the set of relators. We call this the multiplication table presentation.

If $G$ is a group with generators $A$ and relators $R$, then we can view $\widetilde{A}$ as a bouquet of circles labeled by $\widetilde{A}$, that is to say, as a graph $X$ with a single vertex and with edge set $\widetilde{A}$; we impose the equality ordering. Then $\Pi_{1}(X)$ is the free group on $A$ and $G$ is the ordered groupoid presented by $A$ and $R$.

We remark that any ordered groupoid generated by an $(r$-)inductive graph is $(r)$-inductive.

\section{Ordered 2-complexes.}

In the previous section, we saw how to add relations to the fundamental ordered groupoid of an ordered graph; in this section, we look at the geometric analog: Gluing in 2-cells. There are several ways in which we could do this. For now, we shall choose a rather general method; future developments, especially the consideration of higher dimensional complexes, might make it necessary to impose more stringent restrictions.

From now on, we shall be a little less formal about topological ideas (thus requiring a greater familiarity with such on the part of the reader) to avoid the cumbersome task of defining coverings of 2-complexes combinatorially.

We define a 2 -cell to be a regular $n$-gon in $\mathbb{R}^{2}, n>0$, where, by convention, we take a regular 1 -gon to be the unit disk with a single vertex $(0,1)$ and one edge pair $\left\{e, e^{-1}\right\}$, and we take a regular 2-gon to be the unit disk with vertices $(-1,0)$ and $(1,0)$ with the obvious two edge pairs. If $c$ is a 2-cell, its boundary will be denoted $\partial c$.

An ordered 2-complex $X$ consists of an ordered graph $X^{(1)}$ (the 1-skeleton) and a set $C(X)$ consisting of pairs $\left(c, f_{c}\right)$ where $c$ is a 2-cell and $f_{c}: \partial c \rightarrow$ $X^{(1)}$ is a graph morphism called the attaching map for $c$. Topologically, $c$ is glued to $X^{(1)}$ via $f_{c}$. We shall write $V(X)$ and $E(X)$ for the vertex and 
edge sets of $X^{(1)}$. A defining path for $\left(c, f_{c}\right) \in C(X)$ is the image under $f_{c}$ of a simple, nonempty, closed path in $\partial c$. We then require, for any defining path $p$ for $\left(c, f_{c}\right)$ and $w \leq \mathbf{d}(p)$, that $(w \mid p)$ is a defining path for some $\left(c^{\prime}, f_{c^{\prime}}\right) \in C(X)$. Future work may show that we actually want to order $C(X)$ and have a restriction map.

Given an ordered 2-complex, we can define a set of relators by

$$
R_{X}=\left\{p \mid p \text { is a defining path for some }\left(c, f_{c}\right) \in C(X)\right\} ;
$$

the definition of an ordered 2-complex insures that $R_{X}$ is closed under restrictions. We define

$$
\Pi_{1}(X)=\left\langle X \mid R_{X}\right\rangle
$$

to be the fundamental ordered groupoid of $X$. As before, one can verify that two paths in $X^{(1)}$ are homotopic topologically in (the geometric realization of) $X$ if and only if they are equivalent in $\Pi_{1}(X)$; that is, $\Pi_{1}(X)$ embeds naturally as a subgroupoid of the topological fundamental groupoid. Once again, we have the standard (simplicial) fundamental groupoid if we ignore the ordering.

If $v \in V(X)$, then $\pi_{1}(X, v)$ will be used to denote the local group at $v$ and is called the fundamental group of $X$ at $v$. If $Y$ is a connected 2complex, then $\pi_{1}(Y)$ will be used to denote the abstract group to which all fundamental groups of $Y$ are isomorphic. The majority of 2-complexes which we shall consider will not be connected and so we cannot in general speak of the fundamental group of $X$. An ordered 2-complex will be called $\pi_{1}$-trivial if its fundamental group at each vertex is trivial (or, equivalently, each connected component is simply connected).

If $G$ is a group given by generators and relators, then its standard 2complex with the equality ordering has fundamental ordered groupoid exactly the group. More generally, it is immediate how to realize any ordered groupoid given by generators and relators as the fundamental ordered groupoid of an ordered 2-complex.

We shall call an ordered 2-complex $(r$-)inductive if its 1-skeleton is such. In this case, the fundamental groupoid will be $(r$-)inductive as well. It follows that any inverse (regular) semigroup can be represented as the fundamental ordered groupoid of an ordered 2-complex, generalizing the usual result for groups.

Morphisms of ordered 2-complexes are defined in the obvious way. Let $\varphi: X \rightarrow Y$ be a morphism of ordered 2-complexes. Then there is an induced morphism $\varphi_{*}: \Pi_{1}(X) \rightarrow \Pi_{1}(Y)$. If $\varphi$ is a covering morphism in the topological sense, then $\varphi_{*}$ will be a covering morphism, but the converse fails. In general, $\varphi_{*}$ being a, respectively, immersion, fibration, covering is equivalent to $\varphi$ having, respectively, at most one lift to any vertex of each path in $Y$, unique path-lifting, path-lifting. 


\section{The Schützenberger complex.}

In this section, we construct a $\pi_{1}$-trivial covering of an ordered 2-complex which plays a role in the theory analogous to that of the Cayley complex (or universal covering) in group theory. The goal of this section is to prove the following theorem:

Theorem 7.1. Let $X$ be an ordered 2-complex. Then there is a $\pi_{1}$-trivial ordered 2-complex $\widetilde{X}$ and a surjective ordered covering $\varphi: \widetilde{X} \rightarrow X$. Moreover, for each vertex $v \in V(X)$, there is a connected component $\widetilde{X}_{v}$ and a free action of $\pi_{1}(X, v)$ on $\widetilde{X}_{v}$ such that $D_{v}=\pi_{1}(X, v) / \widetilde{X}_{v}$.

Let $X$ be an ordered 2-complex. Then the Schützenberger graph of $X$, denoted $\widetilde{X}^{(1)}$, is defined as follows:

(1) $V\left(\widetilde{X}^{(1)}\right)=E\left(\Pi_{1}(X)\right)$;

(2) $E\left(\widetilde{X}^{(1)}\right)=\left\{(g, x) \mid g \in E\left(\Pi_{1}(X)\right), x \in E(X)\right.$, and $\left.\mathbf{r}(g)=\mathbf{d}(x)\right\}$.

We define $\mathbf{d}(g, x)=g$ and $(g, x)^{-1}=\left(g x, x^{-1}\right)$. The order on $V\left(\widetilde{X}^{(1)}\right)$ is as on $E\left(\Pi_{1}(X)\right)$, while the order on $E\left(\tilde{X}^{(1)}\right)$ is the product order. It is straightforward to check that this is an ordered graph.

For the case of the standard ordered 2-complex of an inverse semigroup presentation (to be defined shortly), the connected components of $\widetilde{X}^{(1)}$ will be the usual Schützenberger graphs of the various $\mathcal{R}$-classes whence the name. In the case that $X$ is the standard 2-complex of a group presentation, $\widetilde{X}^{(1)}$ is the usual Cayley graph.

Lemma 7.2. The ordered graph morphism $\varphi: \widetilde{X}^{(1)} \rightarrow X^{(1)}$ given on vertices by $g \mapsto \mathbf{r}(g)$ and on edges by projection to the second coordinate is a surjective covering.

Proof. It is clear that the map is an ordered graph morphism. To see it is a cover, if $v \in V(X), g \in V\left(\widetilde{X}^{(1)}\right)$ with $\mathbf{r}(g)=v$, and $x \in E(X)$ with $\mathbf{d}(x)=v=\mathbf{r}(g)$, then $(g, x)$ is the unique lift of $x$ to $g$. It follows $\varphi$ is a covering. Surjectivity is clear.

We now define the Schützenberger complex to be the ordered 2-complex with 1-skeleton $\widetilde{X}^{(1)}$ and whose collection of 2-cells consists of all possible lifts of 2-cells of $X$ under the 1-skeleton covering $\varphi$. It is clear $\widetilde{X}$ is an ordered 2-complex and $\varphi$ induces a topological covering. If $X$ is the standard 2-complex of a group presentation, the resulting complex is the Cayley complex of the presentation.

We observe that the connected components of $\widetilde{X}$ correspond to the vertices of $X$; that is, for each $v \in V(X)$, there is a connected component $\widetilde{X}_{v}$ of $\widetilde{X}$ whose vertices consists of $\operatorname{Star}(v)$, and all connected components are of this form. 
If $v, v^{\prime} \in V(X)$ with $v \leq v^{\prime}$, one can check that the restrictions induce an ordered graph morphism from $\widetilde{X}_{v^{\prime}}^{(1)}$ to $\widetilde{X}_{v}^{(1)}$ which can be extended to an ordered 2-complex morphism in a natural way. While this will not be important in our current work, we do mention that it is connected with the notion of an ordered forest as per [25].

We now show that $\widetilde{X}$ is $\pi_{1}$-trivial. Indeed, a path in $\widetilde{X}_{v}$ is of the form

$$
\left(g, x_{1}\right)\left(g x_{1}, x_{2}\right) \ldots\left(g x_{1} \ldots x_{n-1}, x_{n}\right)
$$

where $g \in \Pi_{1}(X)$ with $\mathbf{d}(g)=v$ and $p=x_{1} \ldots x_{n}$ is a path in $X$ with $\mathbf{d}(p)=\mathbf{r}(g)$. To be closed, we must have $g p=g$ in $\Pi_{1}(X)$ whence $p$ is a null homotopic, closed path in $X$. The homotopy killing $p$ can then be lifted to $\widetilde{X}$; it follows $\widetilde{X}$ is $\pi_{1}$-trivial.

Our next goal is to show that $\Pi_{1}(X)$ acts freely on $\widetilde{X}$ and that the quotient can be identified with $X$. In the non-ordered case this is standard, so we don't go into great detail.

Let $G$ be an ordered groupoid and $X$ an ordered 2-complex. Then a left action (analogous to the case of an ordered groupoid acting on an ordered groupoid [21]) $(\pi, A)$ of $G$ on $X$ consists of the following data: First we require an ordered graph morphism $\pi: X^{(1)} \rightarrow \operatorname{Id}(G)$. Now define an ordered 2-complex $(G, X)$ by:

$$
\begin{aligned}
& V(G, X)=\{(g, v) \mid g \in E(G), v \in V(X), \text { and } \mathbf{r}(g)=\pi(v)\} \\
& E(G, X)=\{(g, x) \mid g \in E(G), x \in E(X), \text { and } \mathbf{r}(g)=\pi(x)\} .
\end{aligned}
$$

We let $(g, x) \mathbf{d}=(g, x \mathbf{d})$ and $(g, x)^{-1}=\left(g, x^{-1}\right)$; the order is the product order. The 2-cells are of the form $(g, c)$ where $c$ is a 2-cell of $X$ whose attaching map has image in $\pi^{-1}(\mathbf{r}(g))$. It is straightforward to check that this is an ordered 2-complex. We then require an ordered 2-complex morphism $A:(G, X) \rightarrow X$ (which we normally denote by left multiplication, $A(g, x)=g x)$ such that the following axioms hold (we use $\exists g x$ if $\mathbf{r}(g)=\pi(x))$ :

(A1) If $\exists g x$, then $\pi(g x)=\mathbf{d}(g)$;

(A2) if $\exists g_{1} g_{2}, \exists g_{2} x$, then $g_{1}\left(g_{2} x\right)=\left(g_{1} g_{2}\right) x$;

(A3) $1_{v} x=x, v \in V(G)$, whenever it is defined;

where, in the above, $g, g_{1}, g_{2} \in E(G)$ and $x$ is an $n$-cell of $X, n=0,1,2$. The action is said to be free if $g x=x$ implies $g$ is an identity for such an $x$.

We now define an action of $\Pi_{1}(X)$ on $\widetilde{X}$ by defining $\pi: \widetilde{X} \rightarrow X$ to be the map which takes $\widetilde{X}_{v}$ to $v$. The map $A$ is defined by letting, for $g, g^{\prime} \in$ $E\left(\Pi_{1}(X)\right)$ with $\mathbf{r}(g)=\mathbf{d}\left(g^{\prime}\right), A\left(g, g^{\prime}\right)=g g^{\prime}$. For $g, g^{\prime} \in E\left(\Pi_{1}(X)\right), x \in X$ with $\mathbf{r}(g)=\mathbf{d}\left(g^{\prime}\right)$ and $\mathbf{r}\left(g^{\prime}\right)=\mathbf{d}(x)$, we define $g\left(g^{\prime}, x\right)=\left(g g^{\prime}, x\right)$. The map is extended to 2-cells in the obvious way. This action is clearly free since if $g g^{\prime}=g^{\prime}$, then $g$ is an identity. We leave it to the reader to check the details. 
Observe that if $v \in v(X)$, then $\pi_{1}(X, v)$ acts freely on $\tilde{X}_{v}$. Hence, if this action is co-compact (has compact quotient), $\pi_{1}(X, v)$ is finitely presented.

The following proposition is a standard fact about the action of the fundamental groupoid on the universal cover:

Proposition 7.3. Let $\varphi: \widetilde{X} \rightarrow X$ be the covering projection. Then two $n$-cells are identified by $\varphi$ if and only if they are in the same orbit under the action of $\Pi_{1}(X)$. In fact, if $v \in V(X)$, then $D_{v}=\pi_{1}(X, v) / \widetilde{X}_{v}$.

One can even do better by showing that if we define an order on the quotient complex $\Pi_{1}(X) / \widetilde{X}$ by $[x] \leq\left[x^{\prime}\right]$, with $x \in V(X)$ or $x \in E(X)$, if, for each $y^{\prime} \in\left[x^{\prime}\right]$, there exists $y \in[x]$ such that $y \leq y^{\prime}$ (where we use brackets for orbits), then this order turns the topological homeomorphism of $X$ and $\Pi_{1}(X) / \widetilde{X}$ into an isomorphism of ordered 2-complexes. As we shall not use this fact in the sequel, we leave its verification as a tedious exercise.

\section{The standard ordered 2-complex of an inverse semigroup presentation.}

Let $I=\operatorname{Inv}\langle A \mid R\rangle$ be an inverse semigroup presentation. We now construct an ordered 2-complex $X$ which we call the standard ordered 2-complex of the presentation $\operatorname{Inv}\langle A \mid R\rangle$ of $I$. The vertices of $X$ are $\mathcal{E}(I)$; this makes it clear that our complex will not necessarily be finite for a finite presentation and might not be, in any sense, effectively constructible. The edges are pairs $(e, x)$ where $e \in \mathcal{E}(I), x \in \widetilde{A}$ and ex $\mathcal{R} e$. We define $\mathbf{d}(e, x)=e$ and $(e, x)^{-1}=\left((e x)^{-1} e x, x^{-1}\right)$. We call $x$ the label of $(e, x)$ and extend the notion to paths in the obvious way. If $I$ is a group, then one has a wedge of circles labeled by the elements of $A$ (and their inverses). We define a partial order by $(e, x) \leq(f, y)$ if $e \leq f$ and $x=y$. It is straightforward to see that $X^{(1)}$ satisfies the axioms of an inductive graph. Note that the inverse and restriction maps respect labels. If $A$ is a finite set, then $X^{(1)}$ is locally finite.

Proposition 8.1. Let $e \in V(X)$ and $w \in \widetilde{A}^{*}$ be a word. Then $w$ labels a path starting at $e$ if and only if ew $\mathcal{R} e$ in $I$. The endpoint of such a path is $(e w)^{-1}(e w)$.

Proof. First suppose that $w$ labels such a path; we proceed by induction on $|w|$, the case $|w|=0$ being clear. Suppose $w=u x$ with $x \in \widetilde{A}$ and $u \in \widetilde{A}^{*}$. Since $u$ labels a path starting at $e$, we have, by induction, that $e u \mathcal{R} e$ and the path ends at $f=(e u)^{-1} e u$. It now follows that the last edge of the path labeled by $w$ from $e$ is $(f, x)$ whence $f x \mathcal{R} f$ and the endpoint is $(f x)^{-1} f x$. We can conclude that $u f x \mathcal{R} u f$; but $u f=u u^{-1} e u=e u$ whence we have

$$
\text { ew }=\text { eux } \mathcal{R} \text { eu } \mathcal{R} e .
$$


Also,

$$
\begin{aligned}
(f x)^{-1} f x & =\left(u^{-1} e u x\right)^{-1}\left(u^{-1} e u x\right) \\
& =x^{-1} u^{-1} e u u^{-1} e u x=w^{-1} e w=(e w)^{-1}(e w)
\end{aligned}
$$

and the result follows.

Suppose now that $w$ is such that $e w \mathcal{R}$ e. We induct on $|w|$, the case $|w|=0$ being clear. Suppose $w=u x$ with $x \in \widetilde{A}$ and $u \in \widetilde{A}^{*}$. Then $e \mathcal{R}$ ew $\leq_{\mathcal{R}}$ eu $\leq_{\mathcal{R}}$ e whence $e u \mathcal{R} e$ and so, by induction, $u$ labels a path $p$ from $e$ with endpoint $(e u)^{-1} e u=u^{-1} e u$. But

$$
u^{-1} \text { eux }=u^{-1} e w \mathcal{R} u^{-1} e \mathcal{R} u^{-1} e u
$$

so we can add an edge $\left((e u)^{-1} e u, x\right)$ to $p$ to get a path from $e$ labeled by $w$. One can verify, as above, that the endpoint of $\left((e u)^{-1} e u, x\right)$ is $(e w)^{-1} e w$.

Corollary 8.2. Let $R^{\prime}=\left\{u v^{-1} \mid u=v \in R\right\}$ (note that we do not reduce the word $\left.u v^{-1}\right)$; then every path in $X$ labeled by an element of $R^{\prime}$ is a closed path.

Proof. Suppose $w \in R^{\prime}$ labels a path from a vertex $e$. Then, by Proposition 8.1, ew $\mathcal{R} e$ and the path ends at $(e w)^{-1} e w$. But $w \in \mathcal{E}(I)$, whence ew $\in \mathcal{E}(I)$. Since $\mathcal{R}$-equivalent idempotents are equal, it follows $(e w)^{-1}$ ew $=$ $e w=e$.

We now attach a 2-cell for every path labeled by an element of $R^{\prime}$ (such paths are closed by Corollary 8.2). Clearly the restriction of a defining path is a defining path and so $X$ is an ordered 2-complex. Alternatively, one can describe the 2-cells as follows: For each relation $u=v \in R$, whenever we find a vertex $w$ from which both $u$ and $v$ label a path (in which case both these paths have the same terminus), we add a cell with boundary $u v^{-1}$.

If $I$ is a group, then $X$ is the standard 2-complex of the corresponding group presentation $\left\langle X \mid R^{\prime}\right\rangle$ (again not reducing the elements of $R^{\prime}$ ); here, there may be some spheres with boundaries $x x^{-1}$ or $x^{-1} x$, where $x$ is a generator, as we use the same relations for the inverse semigroup and group presentations).

Viewing $I$ as an inductive groupoid, we can define an ordered graph morphism $\psi: X \rightarrow I$ by the identity on vertices and on edges by $\psi(e, x)=e x$.

Proposition 8.3. The map $\psi$ is an ordered graph morphism. Furthermore, any defining path is sent to an idempotent (that is, a local identity).

Proof. First we show that $\mathbf{d}$ is preserved. Indeed, if $(e, x) \in E(X)$, then ex $\mathcal{R} e$ whence $\mathbf{d}(e x)=e=\mathbf{d}(e, x)$. As to inverses,

$$
\psi\left((e x)^{-1} e x, x^{-1}\right)=x^{-1} e x x^{-1}=x^{-1} x x^{-1} e=x^{-1} e=(e x)^{-1} .
$$

Order is preserved since the natural partial order on an inverse semigroup is compatible with multiplication. An easy induction argument shows that 
a path in $X$, starting at $e$, labeled by $w$, is sent to $e w$. If $p$ is a defining path, then $p$ is labeled by a cyclic conjugate of a word $u v^{-1}$ with $u=v$ or $v=u \in R$. Let $e$ be the vertex in $p$ from which $u v^{-1}$ can be read. Then, by Proposition 8.1, e $\mathcal{R} e u v^{-1}$ whence $e=e u v^{-1}$ since $u v^{-1}$ is an idempotent. Suppose $u v^{-1}=s t$ and the label of $p$ is $t s$. Then, by Proposition 8.1, $p$ starts at $(e s)^{-1} e s=s^{-1} e s$. So $\psi(p)=s^{-1} e s t s=s^{-1} e u v^{-1} s=s^{-1} e s \in \mathcal{E}(I)$ as desired.

It now follows that $\psi$ induces a map $\bar{\psi}: \Pi_{1}(X) \rightarrow I$. We wish to show that this is an isomorphism. Define a map $\tau: A \rightarrow \Pi_{1}(X)$ (where we now view the latter as an inverse semigroup) by $x \mapsto\left(x x^{-1}, x\right)$. If $\tau$ induces a morphism from $I$, then, since $\bar{\psi} \tau$ is the identity on $A$, it would have to induce an isomorphism.

Lemma 8.4. The map induced by $\tau$ from $\widetilde{A}^{+}$to $\Pi_{1}(X)$ takes a word $w$ to the path labeled by $w$ from $w w^{-1}$ to $w^{-1} w$.

Proof. First we observe that, since $w w^{-1} w \mathcal{R} w$, Proposition 8.1 shows that $w$ labels a path from $w w^{-1}$ to $w^{-1} w$. We prove the result by induction, the case $|w|=1$ following from the definition. If $w=u x$ with $u \in \widetilde{A}^{+}$and $x \in \widetilde{A}$, then, by induction, $u$ maps to the path $p$ labeled by $u$ from $u u^{-1}$ to $u^{-1} u$. If $u^{-1} u \mathcal{R} u^{-1} u x$, then

$$
u=u u^{-1} u \mathcal{R} u u^{-1} u x=w,
$$

so there is an edge $\left(u^{-1} u, x\right)$, and $w$ maps to $p\left(u^{-1} u, x\right)$ which is a path from $u u^{-1}=w w^{-1}$ to $\left(u^{-1} u x\right)^{-1} u^{-1} u x=w^{-1} w$ labeled by $w$. If $u^{-1} u x<_{\mathcal{R}}$ $u^{-1} u$, then the image of $w$ is the product of the path labeled by $u$ ending at $u^{-1} u x x^{-1}$ with the edge $\left(u^{-1} u x x^{-1}, x\right)$. But Proposition 8.1 shows that the path labeled by $u^{-1}$ from $u^{-1} u x x^{-1}$ ends at

$$
\left(x x^{-1} u^{-1}\right)^{-1} x x^{-1} u^{-1}=u x x^{-1} u^{-1}=w w^{-1} .
$$

The result follows.

Proposition 8.5. The map $\tau$ induces a morphism from $I$ to $\Pi_{1}(X)$.

Proof. Suppose $u=v \in R$. Let $f=u u^{-1}=v v^{-1}$; then $f=u v^{-1}=f u v^{-1}$. It follows, by Proposition 8.1, that $u v^{-1}$ labels a path from $f$ to $f$ in $X$ which, by construction, is a defining path. Thus the paths from $f$ labeled by $u$ and $v$ exist and are homotopic. But these paths are the images of the words $u$ and $v$ in $\widetilde{A}^{+}$by Lemma 8.4. It follows that $\tau$ induces a morphism.

Corollary 8.6. Let $I=\operatorname{Inv}\langle A \mid R\rangle$ be an inverse semigroup presentation and $X$ the standard ordered 2-complex for this presentation. Then $I=$ $\Pi_{1}(X)$.

Keeping our previous notation, the maximal group image of $I$ is the group $G$ presented by $\left\langle A \mid R^{\prime}\right\rangle$. Furthermore, there is a natural morphism $\psi$ from 
$X$ to the standard 2-complex $Y$ for $G$ with the above presentation (again, we do not reduce the words in $R^{\prime}$ and we may have sphere representing the trivial relations: $x x^{-1}=1=x^{-1} x$ ). This map collapses all the vertices and projects to the second coordinate on edges. The 2-cells are mapped in the natural way. This map is, in fact, an immersion [20] on the 1-skeleton; also, any based 2-cell has at most one based lift to any vertex. McAlister's $P$-theorem $[\mathbf{1 0}]$ can easily be shown to be equivalent to stating that $I$ is $E$-unitary (that is, the natural projection to $G$ is idempotent pure) if and only there is an ordered 2-complex $Z$ containing $X$ and an extension of $\psi$ to $Z$ which is a covering such that $\Pi_{1}(Z)$ is an enlargement of $\Pi_{1}(X)$ in the sense of $[\mathbf{7}, \mathbf{2 1}]$. In fact, all the results of $[\mathbf{2 1}]$ can be restated and proved more generally in the context of ordered 2-complexes where the role of the derived ordered groupoid is replaced by what is called in homotopy theory, the mapping fiber. More on this will appear in a future paper.

We now turn to some examples.

Example 8.7. Let $I$ be the free inverse semigroup given by the presentation $\operatorname{Inv}\langle A \mid\rangle$. Then the standard ordered 2-complex $X$ consists of all finite subtrees of the Cayley graph of a free group on $A$ (with respect to generators A) containing 1 as a vertex (called Munn trees). We will denote a vertex by a pair $(T, g)$ where $T$ is a Munn tree and $g \in G$ labels a vertex of $T$. Edges will be denoted by triples $(T, g, x)$ where $T$ is a Munn tree, $g \in G$ is a vertex of $T$, and $x \in A$ labels an edge in $T$ from $g$. The domain of $(T, g, x)$ is $(T, g),(T, g, x)^{-1}=\left(T, g x, x^{-1}\right)$. The order on $V(X)$ is given by $(T, g) \leq\left(T^{\prime}, g^{\prime}\right)$ if and only if $g=g^{\prime}$ and $T^{\prime} \subseteq T$. The order on $E(X)$ is given by $(T, g, x) \leq\left(T^{\prime}, g^{\prime}, x^{\prime}\right)$ if and only if $g=g^{\prime}, x=x^{\prime}$, and $T^{\prime} \subseteq T$. Note that $X$ is $\pi_{1}$-trivial.

Example 8.8. A Brandt semigroup is an inverse semigroup whose underlying set is $(J \times G \times J) \cup 0$, with $G$ a group and $J$ a set, and whose nonzero multiplications are given by

$$
(i, g, j)\left(j, g^{\prime}, i^{\prime}\right)=\left(i, g g^{\prime}, i^{\prime}\right) .
$$

This inverse semigroup will be denoted $\mathcal{M}^{0}(J, G)$. Suppose $J$ has a distinguished element $j_{1}$. If $G=\langle A \mid R\rangle$ is a presentation for $G$, we can obtain a presentation $\mathcal{M}^{0}(J, G)$ by taking as generators $J \backslash j_{1} \cup A \cup z$ where, for $j \in J \backslash j_{1}, j \mapsto\left(j, 1, j_{1}\right)$, for $a \in A, a \mapsto\left(j_{1}, a, j_{1}\right)$, and $z$ maps to 0 . Choose a distinguished element $a_{0} \in A$. For relations, we use: The relations of $R$ but where 1 is replaced by $a_{0} a_{0}^{-1}$; the relations $j^{-1} j=a_{0} a_{0}^{-1}, j \in J \backslash j_{1}$; $j^{-1} j^{\prime}=z$ if $j \neq j^{\prime} \in J \backslash j_{1} ; x j=z, x \in A \cup J \backslash j_{1} ; a a^{-1}=a^{-1} a, a \in A$; $a a^{-1}=a_{0} a_{0}^{-1}, a \in A$; and the relations needed to make $z$ a zero. The standard ordered 2-complex $X$ has two connected components. One component has a single vertex 0 and edges which are loops labeled by $A \cup J \backslash j_{1} \cup z$. The 2-cells give us the standard 2-complex for $G$ plus some 2-cells with 
contractible defining paths and some 2-cells with defining paths homotopic to the various edges and their conjugates by $z$ (whence the local group is trivial). The other component has vertices of the form $(j, 1, j)$ with $j \in J$. At the vertex $\left(j_{1}, 1, j_{1}\right)$ there is a copy of the standard 2-complex of $G$ plus some 2-cells with contractible defining paths. For $j \in J \backslash j_{1}$, there is an edge from $(j, 1, j)$ to $\left(j_{1}, 1, j_{1}\right)$ labeled by $j$. There are also 2 -cells with contractible defining paths labeled by $j^{-1} j a_{0} a_{0}^{-1}$. This connected component is clearly homotopy-equivalent to a wedge of the standard 2-complex for $G$ and some spheres, so the local group is $G$.

Example 8.9. Consider the bicyclic semigroup $I=\operatorname{Inv}\left\langle x \mid x x x^{-1}=x\right\rangle$. One can check that standard ordered 2-complex has 1-skeleton: The interval $[0, \infty)$ with its usual graph structure; the vertices are ordered by $\geq$; the edges are incomparable. Each interval of length two is the seam glued of an attached American football. Thus the complex is homotopy-equivalent to a countably infinite wedge of spheres, and hence has trivial fundamental group.

Let $X$ be the standard ordered 2-complex of an inverse semigroup presentation $I=\operatorname{Inv}\langle A \mid R\rangle$. We now consider the 1-skeleton of the Schützenberger complex $\tilde{X}$. The vertices are the elements of $I$. Edges are of the form $(s,(e, x))$ where $s^{-1} s=e$ and $(e, x)$ is an edge of $X$. Thus edges can be more succinctly described as pairs $(s, x)$ with $s \in I$ and $s^{-1} s x \mathcal{R} s^{-1} s$ or, equivalently, sx $\mathcal{R} s ; \mathbf{d}(s, x)=s,(s, x)^{-1}=\left(s x, x^{-1}\right)$. Connected components correspond to $\mathcal{R}$-classes and 2-cells are added whenever an element of $R^{\prime}$ labels a path. The reader familiar with the work of Stephen [24] will see immediately that the 1-skeleton of the connected component corresponding to an $\mathcal{R}$-class is the Schützenberger graph of that $\mathcal{R}$-class. Stephen's ToddCoxeter-like procedure to construct the Schützenberger graph can easily be modified to construct the corresponding component of the Schützenberger complex by adding a 2-cell every time an expansion is performed. We note that the left action of $I$ on $\widetilde{X}$ is "essentially" the left Preston-Wagner representation [7].

The covering $\varphi: \widetilde{X} \rightarrow X$ takes a vertex $s$ to $s^{-1} s$ and takes the connected component of $\widetilde{X}_{e}$ corresponding to an idempotent $e$ onto the $\mathcal{D}$-class $D_{e}$ of $X$ corresponding to the $\mathcal{D}$-class of $e$ in $I$. Two elements of the $\mathcal{R}$-class of $e$ get identified if and only if they are $\mathcal{H}$-equivalent. In fact, $H_{e}$, the $\mathcal{H}$-class of $e$, acts freely on the left of $\widetilde{X}_{e}$ and the quotient is $D_{e}$ by Proposition 7.3. Thus we have the following result:

Theorem 8.10. Suppose that $I$ is a finitely generated (presented) inverse semigroup and $H_{e}$ is a maximal subgroup with identity e such that the left action of $H_{e}$ on its $\mathcal{R}$-class $R_{e}$ has finitely many orbits. Then $H_{e}$ is finitely generated (presented). 
Proof. Let $X$ be the standard ordered 2-complex and $D_{e} \subseteq X$ the $\mathcal{D}$-class of $e$. Our above discussion shows that $V\left(D_{e}\right)=H_{e} / R_{e}$ and hence is finite. Since $I$ is finitely generated, it follows that $D_{e}^{(1)}$ is a finite vertex, locally finite graph: That is, $D^{(1)}$ is finite. Thus $H_{e}=\pi_{1}\left(D_{e}\right)$ is finitely generated. If $I$ is also finitely presented, then $R^{\prime}$ (defined as above) is finite. Since $D_{e}^{(1)}$ is also finite, we can only find finitely many paths in $D_{e}$ labeled by elements of $R^{\prime}$ whence, by construction of $X, D_{e}$ is a finite 2-complex. It follows $H_{e}=\pi_{1}\left(D_{e}\right)$ is finitely presented.

It is a simple exercise to give an explicit presentation of $H_{e}$ using the standard techniques for computing the fundamental group of a finite 2complex. Similar results were obtained in [17] for semigroup presentations of regular semigroups using Reidemeister-Schreier-type rewriting systems.

This result leads us to define a finitely generated inverse semigroup to be hyperbolic if $\widetilde{X}$ is a Gromov hyperbolic (disconnected) space, and all the maximal subgroups act co-compactly on the corresponding component of $\widetilde{X}$. This would be a generalization of hyperbolic groups and an immediate consequence of the definition is that all the maximal subgroups are hyperbolic (since any group acting properly discontinuously and co-compactly on a hyperbolic space is hyperbolic). Similarly, we define a finitely presented inverse semigroup to be Fuchsian if its Schützenberger complex is planar (after identifying 2-cells which give rise to the same defining paths, and removing 2-cells with contractible defining paths), and each maximal subgroup acts co-compactly on the corresponding component of $\widetilde{X}$. Once again, this implies that the maximal subgroups are Fuchsian. These classes will be studied in future papers; also notions of Van Kampen diagrams over inverse semigroup presentations will be introduced in future work.

We end this section with the following observation: If $D$ is a $\mathcal{D}$-class of $I$, then $I$ acts on the right of the set of $\mathcal{L}$-classes of $D$ by partial bijections. The transition inverse semigroup is what Rhodes calls the right letter mapping semigroup of $I$ corresponding to $D[6]$. One can then form the right letter mapping graph of $D$ whose vertices are the $\mathcal{L}$-classes of $D$ and whose edges are of the form $(b, x)$ whenever $b x$ is defined in the right letter mapping transformation semigroup. One has $\mathbf{d}(b, x)=b$ and $(b, x)^{-1}=\left(b x, x^{-1}\right)$. It is straightforward to verify that the map taking $b$ to the unique idempotent in $b$ is an isomorphism of the right letter mapping graph with the $\mathcal{D}$-class of $X$ corresponding to $D$. The hypothesis of Theorem 8.10 can be rephrased in terms of asking that the right letter mapping transformation semigroup of the $\mathcal{D}$-class of $e$ be finite. Since the Munn representation is the direct sum of the right letter mapping representations, $X^{(1)}$ can be viewed as the graph of the Munn representation. Similarly, $\widetilde{X}^{(1)}$ can be view as the graph of the right Preston-Wagner representation which is the direct sum of the Schützenberger representations. 


\section{Amalgams.}

In this section, we study amalgams of the various structures we have been considering.

9.1. Amalgams of partially ordered sets. An amalgam of partially ordered sets is a triple of partially ordered sets $(S, U, T)$ with $S \cap T=U$. The amalgamated product $S \coprod_{U} T$ is the partially ordered set $S \cup T$ with order the union of the orders of $S$ and $T$ and, in addition, for $s \in S$ and $t \in T$, one defines $s \leq t$ if there exists $u \in U$ with $s \leq u \leq t$ (note that if $s$, respectively, $t$ is in $U$, this situation occurs if and only if $s \leq t$ in $S$, respectively, $T$ ); $t \leq s$ is defined similarly.

Proposition 9.1. $S \coprod_{U} T$ is a partially ordered set and, given any partially ordered set $P$ and morphisms $\varphi: S \rightarrow P, \psi: T \rightarrow P$, agreeing on $U$, there is a unique morphism $\tau: S \coprod_{U} T \rightarrow P$ extending $\varphi$ and $\psi$.

Proof. For anti-symmetry, it suffices to show that, for $s \in S$ and $t \in T, s \leq t$ and $t \leq s$ implies $s=t$. Indeed, we then have $u, u^{\prime} \in U$ with $s \leq u \leq t$ and $t \leq u^{\prime} \leq s$; so $s \leq u \leq u^{\prime} \leq s$ and $u \leq t \leq u^{\prime}$ whence $s=u=u^{\prime}=t$. As for transitivity, the only cases to deal with (up to exchanging the role of $s$ and $t$ ) are: $s \leq s^{\prime}, s^{\prime} \leq t ; s \leq t, t \leq t^{\prime}$; and $s \leq t, t \leq s^{\prime}$ where $s, s^{\prime} \in S$ and $t, t^{\prime} \in T$. In the first case, we have $s \leq s^{\prime} \leq u \leq t$ for some $u \in U$ whence $s \leq t$; the second case is similar. For the third case, we have $u, u^{\prime} \in U$ such that $s \leq u \leq t$ and $t \leq u^{\prime} \leq s^{\prime}$ whence $u \leq u^{\prime}$ and $s \leq u \leq u^{\prime} \leq s^{\prime}$.

Now set $\tau=\varphi \cup \psi$. To see that $\tau$ preserves order, it is enough, without loss of generality, to show that, for $s \in S, t \in T$, if $s \leq t$ then $\tau(s) \leq \tau(t)$. But in this case, we have $u \in U$ with $s \leq u \leq t$ whence

$$
\tau(s)=\varphi(s) \leq \varphi(u)=\psi(u) \leq \psi(t)=\tau(t)
$$

and the result follows.

Proposition 9.2. Suppose $(S, U, T)$ is a partially ordered set amalgam with $U$ an order ideal of $S$ and $T$. Then $S \coprod_{U} T$ is just $S \cup T$ with the union of the two partial orders, and $S, T$, and $U$ are order ideals.

Proof. We first prove the statement about order ideals. Suppose $x \leq s \in S$ and $x \in T$. Then there exists $u \in U$ with $x \leq u \leq s$ and so $x \in U \subseteq S$. Thus $S$ and, by a dual argument, $T$ are order ideals. Since $U=S \cap T, U$ is also an order ideal. It now follows immediately that the order is just the union of the orders.

We now give a condition under which the partially ordered set amalgamation of a semilattice amalgam is a semilattice.

Proposition 9.3. Suppose $(S, U, T)$ is a partially ordered set amalgam with $U$ an order ideal of $S$ and $T$. Suppose, further, that $S$ and $T$ are semilattices and that $U$ has a maximum e. Then $S \coprod_{U} T=S \cup T$ is a semilattice. 
Proof. Note first that, since $U$ is an order ideal in $S$ and $T$, it is a semilattice. By Proposition 9.2, it suffices to show that if $s \in S$ and $t \in T$, then $s$ and $t$ have a meet. We claim that the meet is $(s \wedge e) \wedge(e \wedge t)$; this element is well-defined since $U$ is an order ideal in $S$ and $T$. Indeed, any common lower bound $g$ is in $S \cap T=U$. Thus $g \leq s, t$, e whence $g \leq s \wedge e, e \wedge t \in U$ and the result follows.

9.2. Amalgams of ordered 2-complexes. An amalgam of ordered 2complexes consists of a triple of ordered 2-complexes $(X, Y, Z)$ with $Y=$ $X \cap Z$. The amalgamated product $X \coprod_{Y} Z$ is defined to be the universal ordered 2-complex with morphisms of $X$ and $Z$ into it agreeing on $Y$ (if such exists).

Suppose $(X, Y, Z)$ is an amalgam of ordered 2-complexes. Then $X \cup Z$ has the natural structure of a 2-complex with ordered vertex and edge sets; that is:

(1) $V(X \cup Z)=V(X) \coprod_{V(Y)} V(Z)$;

(2) $E(X \cup Z)=E(X) \coprod_{E(Y)} E(Z)$;

(3) $C(X \cup Z)=C(X) \cup C(Y)$.

There are naturally induced, order-preserving maps $\mathbf{d}$ and $e \mapsto e^{-1}$ by the universal property of the amalgamated product of partially ordered sets (also $e \neq e^{-1}$ if such is true for $X$ and $Z$ ). We call this the natural order structure on $X \cup Z$.

We say an amalgam of ordered 2-complexes is tame if $X \cup Z$ with the natural order structure is an ordered 2-complex. It is immediate that in this case $X \coprod_{Y} Z=X \cup Z$. We now give some examples of tame amalgams.

Proposition 9.4. Suppose $(X, Y, Z)$ is an ordered graph amalgam such that $V(Y)$ is an order ideal of $V(X)$ and $V(Z)$. Then $(X, Y, Z)$ is tame.

Proof. We first show that $(X \cup Y)^{(1)}$ is an ordered graph. Suppose $v \leq w$ and $e \in \operatorname{Star}(w)$; we must show there is a unique edge $e^{\prime} \leq e$ in $\operatorname{Star}(v)$. Without loss of generality, assume $e \in E(X)$ (and so $w \in V(X)$ ). Then, since $V(X)$ is an order ideal by Proposition 9.2, $v \in V(X)$ and $(v \mid e)$ is one such edge. Suppose $e^{\prime}$ is another such (necessarily $v \in V(Y)$ and $e^{\prime} \in E(Z)$ ). But then there is an edge $e^{\prime \prime} \in E(Y)$ with $e^{\prime} \leq e^{\prime \prime} \leq e$, so $e^{\prime}=\left(v \mid e^{\prime \prime}\right) \in E(Y) \subseteq E(X)$ and $e^{\prime} \leq e$ whence $e^{\prime}=(v \mid e)$ as desired. Note that the above argument shows that $E(X)$ and $E(Z)$ are order ideals in $E(X \cup Z)$.

Assume now that $p$ is a defining path of $\left(c, f_{c}\right) \in C(X) \cup C(Y)$ and $v \leq$ $\mathbf{d}(p)$. Without loss of generality, assume $\left(c, f_{c}\right) \in C(X)$. Then $v \in V(X)$ (since $V(X)$ is an order ideal), and the restriction $(v \mid p)$ in $X$ and in $X \cup Z$ coincide whence $(v \mid p)$ is a defining path of a 2-cell in $X$.

In particular, all amalgams of unordered groupoids are tame.

An ordered subcomplex (subgroupoid) $Y$ of an ordered 2-complex (groupoid) $X$ is said to be $\mathcal{D}$-saturated in $X$ if it is a union of $\mathcal{D}$-classes of $X$. 
Proposition 9.5. Let $(X, Y, Z)$ be an amalgam of inductive groupoids such that $Y$ is $\mathcal{D}$-saturated in both $X$ and $Z$. Then $(X, Y, X)$ is tame.

Proof. We begin by showing that $(X \cup Y)^{(1)}$ is an ordered graph. Suppose, without loss of generality, that $e \in E(X)$ and $v \leq \mathbf{d}(e)$. If $v \in V(X)$, then $(v \mid e) \leq e$ and if $f \leq e$ with $\mathbf{d}(f)=v$ and $f \neq(v \mid e)$, then $f \in E(Z)$ and $v \in V(Y)$. But $Y$ is $\mathcal{D}$-saturated in $Z$, so $f \in E(Y)$ whence $f=(v \mid e)$ proving uniqueness in this case. Suppose now $v \in V(Z) \backslash V(Y)$, then there exists $w \in V(Y)$ with $v \leq w \leq \mathbf{d}(e)$. Then, since $Y$ is $\mathcal{D}$-saturated in $X$, $(w \mid e) \in Y$ whence $f=(v \mid(w \mid e)) \leq e$ and $\mathbf{d}(f)=v$. Suppose now that $f^{\prime} \leq e$ with $\mathbf{d}\left(f^{\prime}\right)=v$. Then $f^{\prime} \in E(Z)$ and there exists $y \in E(Y)$ with $f^{\prime} \leq y \leq e$. Let $u=w \wedge \mathbf{d}(y) \in V(Y)$. Then $(u \mid y)=(u \mid e)=(u \mid(w \mid e))$ whence

$$
f^{\prime}=(v \mid y)=(v \mid(u \mid e))=(v \mid(w \mid e))
$$

and we have uniqueness.

Now suppose $p$ is the defining path of $\left(c, f_{c}\right) \in C(X) \cup C(Z)$; without loss of generality, we take $\left(c, f_{c}\right) \in C(X)$. Suppose $v \leq \mathbf{d}(p)$. If $v \in V(X)$, then $(v \mid p)$ is the same in $X$ and $X \cup Z$, so it is a defining path of some 2-cell in $C(X)$. Suppose now $v \in V(Z) \backslash V(Y)$. Then there exists $w \in V(Y)$ with $v \leq w \leq \mathbf{d}(p)$. Since $Y$ is $\mathcal{D}$-saturated in $X,(w \mid p)$ is in $Y$ and is the defining path of a 2-cell of $X$ which must, in fact, belong to $Y$ since $Y$ is $\mathcal{D}$-saturated. Then $(v \mid(w \mid p))$ is the defining path of a 2-cell in $Z$.

9.3. Graphs of groups. To study the maximal subgroups of an amalgamated product of ordered groupoids, we need to recall the notion of a graph of groups [19]. A graph of groups $(\mathcal{G}, X)$ consists of a graph $X$ and an assignment to each vertex $v$ of a group $G_{v}$, to each pair of edges $\left\{e, e^{-1}\right\}$ of a group $G_{e}=G_{e^{-1}}$, and to each edge $e$ an inclusion $\iota_{e}: G_{e} \rightarrow G_{\mathbf{d}(e)}$. If $(\mathcal{G}, X)$ is a connected graph of groups and $T$ is a maximal subtree of $X$, then fundamental group of $(\mathcal{G}, X)$ with respect to the tree $T$ is

$$
\begin{gathered}
\pi_{1}(\mathcal{G}, X)=\left\langle G_{v}(v \in V(X)), y_{e}(e \in E(X))\right| y_{t}=1(t \in T), \\
\left.y_{e}^{-1}=y_{e^{-1}}(e \in E(X)), y_{e}^{-1} \iota_{e}(g) y_{e}=\iota_{e^{-1}}(g)\left(e \in E(X), g \in G_{e}\right)\right\rangle .
\end{gathered}
$$

This group is independent of the choice of $T[\mathbf{1 9}]$.

A graph of 2-complexes $(\mathcal{C}, X)$ consists of a graph $X$ and an assignment to each vertex $v$ of a 2-complex $X_{v}$ (called a vertex complex), to each pair of edges $\left\{e, e^{-1}\right\}$ of 2-complex (called an edge complex) $X_{e}=X_{e^{-1}}$, and to each edge $e$ an inclusion $\iota_{e}: X_{e} \rightarrow X_{\mathbf{d}(e)}$. The realization of $(\mathcal{C}, X)$ is obtained by replacing each vertex $v$ by $X_{v}$ and each pair of edges $\left\{e, e^{-1}\right\}$ by $X_{e} \times[0,1]$; one then glues, for each pair of edges $\left\{e, e^{-1}\right\}, X_{e} \times 0$ to the copy of $X_{e}$ in $X_{\mathbf{d}(e)}$ and $X_{e} \times 1$ to the copy in $X_{\mathbf{r}(e)}$. In general, we say that a graph of 2-complexes $X$ gives a graph of 2-complexes decomposition of a 2-complex $Y$ if $Y$ is homotopy-equivalent to the realization of $(\mathcal{C}, X)$. 
A simple application of the Seifert-Van Kampen Theorem shows that if $X$ is the geometric realization of a connected graph of connected 2-complexes, then $\pi_{1}(X)$ is isomorphic to the fundamental group of the graph of groups obtained by replacing each vertex and edge complex by its fundamental group. Conversely, the fundamental group of any connected graph of groups can be so realized.

The following simple observation, which we state as a proposition, will be key to studying the structure of maximal subgroups of amalgams:

Proposition 9.6. Let $(\mathcal{C}, X)$ be a graph of 2 -complexes. Then its realization has the following decomposition as a graph $\left(\mathcal{C}^{\prime}, Y\right)$ of connected 2 -complexes: $V(Y)$ is the set of $\mathcal{D}$-classes of the vertex complexes of $X ; E(Y)$ is the set of $\mathcal{D}$-classes of the edge complexes of $X$; if $e \in E(X)$ and $Z$ is a $\mathcal{D}$-class of $X_{e}$, then $Z$ is a subcomplex of a unique $\mathcal{D}$-class of $X_{\mathbf{d}(e)}$ which we define to be the domain of the edge corresponding to $Z$; the inverse of the edge corresponding to $Z$ is the copy of $Z$ in $X_{e^{-1}}$; and the vertex (edge) complex corresponding to a $\mathcal{D}$-class is the $\mathcal{D}$-class itself.

We illustrate the above proposition in Figure 1 where we have a segment of disconnected complexes as this is the case of interest.

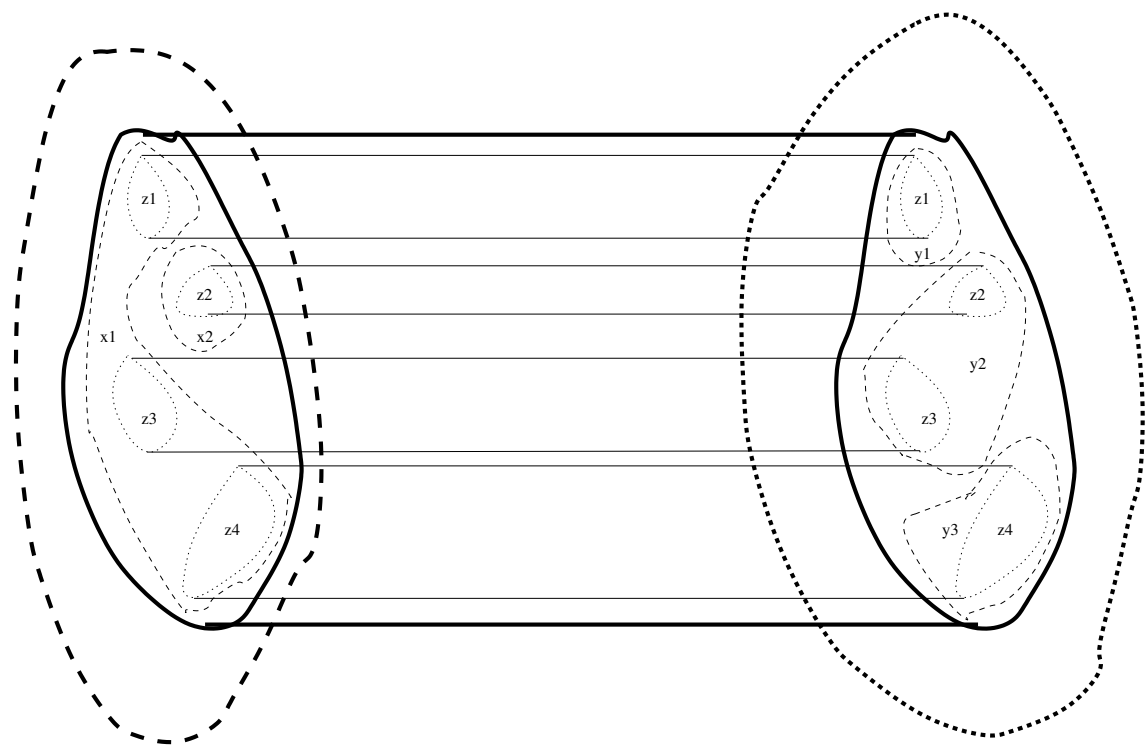

Figure 1. An illustration of Proposition 9.6.

Here, the left vertex complex (surrounded in large thick dashes) has two $\mathcal{D}$-classes $x 1$ and $x 2$ (dashed lines); the right vertex complex (surrounded in small thick dashes) has three $\mathcal{D}$-classes $y 1, y 2$, and $y 3$ (dashed lines); the edge complex (surrounded in thick solid lines) has four $\mathcal{D}$-classes $z 1$, 
$z 2, z 3$, and $z 4$ (dotted lines). The resulting decomposition is a connected, bipartite graph of connected 2-complexes with five vertices and four edges (see Figure 2); this will always be the case for a segment of 2-complexes although in general the bipartite graph will not be connected.

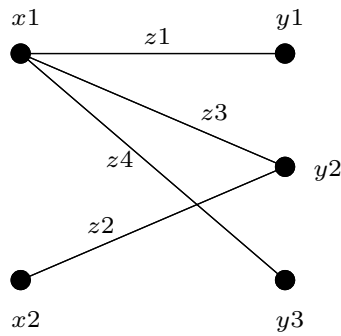

Figure 2. The underlying graph in Figure 1.

Proposition 9.6 gives us a means to obtain a graph of groups decomposition of any maximal subgroup of the fundamental groupoid of a 2-complex with a graph of 2-complexes decomposition. The main such decomposition of interest in the is paper is the following:

Proposition 9.7. Let $(X, Y, Z)$ be a tame amalgam of ordered 2-complexes. Then $X \coprod_{Y} Z$ is homotopy-equivalent to the realization of the graph of 2 complexes $(\mathcal{C}, W)$ whose underlying graph is a segment with $X$ as the left vertex complex, $Z$ as the right vertex complex, and $Y$ as the edge complex.

Proof. By assumption $X \coprod_{Y} Z=X \cup Z$. The homotopy equivalence is induced by applying to the realization of $(\mathcal{C}, W)$ the contraction of $Y \times[0,1]$ to $Y \times 0$.

9.4. Amalgams of ordered groupoids. An amalgam of ordered groupoids consists of a triple $(S, U, T)$ such that $U=S \cap T$. The amalgamated product $S \coprod_{U} T$ is then the universal object with maps of $S$ and $T$, agreeing on $U$, into it (if such exists). Our goal is to study topologically the structure of the maximal subgroups of the amalgamated product. Our results generalize those of $[\mathbf{3}, \mathbf{1 2}]$ for full amalgams of regular semigroups, but our techniques are different.

If $(S, U, T)$ is an ordered groupoid amalgam, we define a graph of groups $\left(\mathcal{G}_{(S, U, T)}, \Gamma\right)$ as follows: $V(\Gamma)$ is the union of the set of $\mathcal{D}$-classes of $S$ and the set of $\mathcal{D}$-classes of $T ; E(\Gamma)$ is the set of $\mathcal{D}$-classes of $U$ (and formal inverses of such); each $\mathcal{D}$-class of $U$ is contained in a unique $\mathcal{D}$-class of $S$, which we take as the initial vertex of the corresponding edge, and a unique $\mathcal{D}$-class of $T$, which we take as its final vertex; we associate to each vertex and edge the maximal subgroup of the corresponding $\mathcal{D}$-class with the obvious inclusion maps (cf. [3]). If $v \in V(S) \cup V(T)$, we let $\left(\mathcal{G}_{v}, \Gamma_{v}\right)$ be the connected component of $\left(\mathcal{G}_{(S, U, T)}, \Gamma\right)$ containing the $\mathcal{D}$-class of $v$. 
We say an ordered 2-complex amalgam $\left(X_{1}, X_{2}, X_{3}\right)$ represents an ordered groupoid amalgam $\left(S_{1}, S_{2}, S_{3}\right)$ if $\Pi_{1}\left(X_{i}\right)=S_{i}, i=1,2,3$. Abstract nonsense shows that if $X_{1} \coprod_{X_{2}} X_{3}$ exists, then $S_{1} \coprod_{S_{2}} S_{1}=\Pi_{1}\left(X_{1} \coprod_{X_{2}} X_{3}\right)$. We say that an ordered groupoid amalgam is tame if it can be represented by a tame ordered 2-complex amalgam.

Theorem 9.8. Suppose $(S, U, T)$ is a tame ordered groupoid amalgam. Then $S \coprod_{U} T$ exists, has vertex set $V(S) \cup V(T)$, and the maximal subgroup corresponding to a vertex $v$ is $\pi_{1}\left(\mathcal{G}_{v}, \Gamma_{v}\right)$.

Proof. Choose a tame ordered 2-complex amalgam $(X, Y, Z)$ representing $(S, U, T)$. Then, $X \coprod_{Y} Z=X \cup Z$ has a graph of 2-complexes decomposition as per Proposition 9.7 whence Proposition 9.6 shows that the maximal subgroups are as described in the theorem statement.

Note that the theorem always applies if the groupoids are unordered.

Corollary 9.9. Suppose $(S, U, T)$ is a tame ordered groupoid amalgam such that $U$ has only trivial subgroups. Then the local groups of $S \coprod_{U} T$ are free products of local groups of $S$, local groups of $T$, and free groups. In particular, if all the local groups of $S$ and $T$ are trivial, then the subgroups of $S \coprod_{U} T$ are free.

Proof. The fundamental group of a connected graph of groups in which all edge groups are trivial is a free product of vertex groups and a free group.

Proposition 9.10. Let $(S, U, T)$ be an ordered groupoid amalgam. Suppose that either $V(U)$ is an order ideal of $V(S)$ and $V(T)$, or $U$ is $\mathcal{D}$-saturated in $S$ and $T$, and $S, U$, and $T$ are inductive. Then $(S, U, T)$ is tame.

Proof. Let $X, Y, Z$ be the multiplication table presentations of, respectively, $S, U, T$. Then $(X, Y, Z)$ represents $(S, U, T)$. In the case that $V(U)$ is an order ideal, we see that $(X, Y, Z)$ is tame by Proposition 9.4, while in the case that $V(U)$ is $\mathcal{D}$-saturated and the amalgam consists of inductive groupoids, the result follows from Proposition 9.5.

Note that if $S, U$, and $T$ are inductive and $U$ is $\mathcal{D}$-saturated, then the graph of groups $\left(\mathcal{G}_{(S, U, T)}, \Gamma\right)$ consists of isolated vertices corresponding to $\mathcal{D}$-classes not in $U$ and disjoint segments corresponding to $\mathcal{D}$-classes in $U$; the inclusions are isomorphisms for these segments. It follows that if $v \in$ $V(S) \cup V(T)=V\left(S \coprod_{U} T\right)$, then the maximal subgroup at $v$ is unchanged on forming the amalgamated product.

9.5. Amalgams of inverse and regular semigroups. We now wish to apply the above theory to inverse and regular semigroup amalgams. If $(S, U, T)$ is an amalgam of inverse (regular) semigroups, we use $S *_{U} T$ for the amalgamated product. In the case of inverse semigroup amalgams $(S, U, T)$, a well-known theorem of Hall [4] shows that $S$ and $T$ embed in 
$S *_{U} T$ in such a manner that their intersection is $U$. He later generalized this to full regular semigroup amalgams [5] (that is, amalgams $(S, U, T)$ such that $\mathcal{E}(S)=\mathcal{E}(U)=\mathcal{E}(T)$.

The following generalizes results of $[\mathbf{3}, \mathbf{1 2}]$ where the case of full amalgams is considered; see [3] for examples; other generalizations (some of which we shall obtain below) can be found in [1].

Theorem 9.11. Suppose $(S, U, T)$ is an inverse (regular) semigroup amalgam with $\mathcal{E}(S)=\mathcal{E}(U)$ an order ideal of $\mathcal{E}(T)$. Then $S *_{U} T=S \coprod_{U} T$, and the maximal subgroup corresponding to an idempotent $e \in \mathcal{E}\left(S *_{U} T\right)=$ $\mathcal{E}(S) \cup \mathcal{E}(T)$ is $\pi_{1}\left(\mathcal{G}_{e}, \Gamma_{e}\right)$. In particular, if $S$ and $T$ only have trivial subgroups, then the subgroups of $S *_{U} T$ are free.

Proof. Since $\mathcal{E}(U)$ is an order ideal in $\mathcal{E}(S)$ and $\mathcal{E}(T)$, Proposition 9.10 shows $(S, U, T)$ is tame whence Theorem 9.8 applies. Since

$$
V\left(S \coprod_{U} T\right)=V(S) \cup V(T)=V(T)
$$

is a semilattice (biordered set), $S \coprod_{U} T$ is an $(r$-)inductive groupoid. Suppose $\varphi: S \rightarrow I$ and $\psi: T \rightarrow I$ are homomorphisms agreeing on $U$ where $I$ is an inverse (regular) semigroup. Then the induced ordered groupoid map $\tau: S \coprod_{U} T \rightarrow I$ agrees with $\psi$ on $V(T)=V\left(S \coprod_{U} T\right)$ and therefore preserves the semilattice (biordered) structure and so $\tau$ is a homomorphism of inverse (regular) semigroups.

The above theorem applies, in particular, to the case of full amalgams. Assume $(S, U, T)$ is a regular semigroup amalgam satisfying the hypotheses of Theorem 9.11. If $U$ has only trivial subgroups, then Corollary 9.9 and the Kurosh Theorem [8] imply that the subgroups of $S *_{U} T$ are isomorphic to free products of free groups and subgroups of the factors $S$ and $T$. Work of Ordman $[\mathbf{1 3}, \mathbf{1 4}]$ gives normal forms for amalgamated products of unordered groupoids. Since the above theorem implies that the underlying groupoid of $S *_{U} T$ is the amalgamated product in the category of unordered groupoids, his results (combined with our description of the order) can be used to completely understand $S *_{U} T$. Also, standard results on groupoid amalgams $[\mathbf{1 3}, \mathbf{1 4}]$ show that $S$ and $T$ embed in $S *_{U} T=S \coprod_{U} T$ with intersection $U$. Since the inclusions are the identity on vertices, they preserve the $(r$-)inductive structure. Thus we have the following generalization of a theorem of Hall [5]:

Theorem 9.12. Let $(S, U, T)$ be a regular semigroup amalgam with $\mathcal{E}(U)=$ $\mathcal{E}(S)$ an order ideal in $\mathcal{E}(T)$. Then $S$ and $T$ embed in $S{ }{ }_{U} T$ with intersection $U$. 
An illustrative example is the case where $S=\operatorname{Inv}\left\langle x \mid x^{3}=0\right\rangle, T$ is a copy of the subsemigroup of $S$ generated by $x^{2}$, made disjoint off the idempotents, and $U=E(T)$. Note that $U$ is an order ideal in $\mathcal{E}(S)$. Using the multiplication table presentation, $S$ can be represented by a 2 -complex with three components: The top component is a segment of length 1 ; the middle component is a segment of length 2 ; and the bottom component is a regular 1-cell. One can similarly represent $T$, only one drops the top component. Each of the original semigroups is finite with trivial subgroups. Then $S *_{U} T$ is represented by identifying the two bottom components and gluing the two middle components along the vertices. It follows that the maximal subgroup corresponding to the middle component is free of rank 2 and the other maximal subgroups are trivial. We invite the reader to construct $(\mathcal{G}, \Gamma)$ and verify that the answer obtained in this manner coincides.

We are now in a position to prove some more difficult results on amalgams which coincide in part with results of [1]. Fix a tame inverse semigroup amalgam $(S, U, T)$. The inclusions of $S$ and $T$ induce a natural ordered groupoid morphism $\varphi: S \coprod_{U} T \rightarrow S *_{U} T$. Our goal is to show that this map is an embedding of $S \coprod_{U} T$ as a $\mathcal{D}$-saturated ordered subgroupoid.

Lemma 9.13. Let $(S, U, T)$ be a tame amalgam of inductive groupoids. Then the inclusions of $S$ and $T$ into $S \coprod_{U} T$ are embeddings. Furthermore, if e, $f \in \mathcal{E}(S)$, then ef is the meet of $e$ and $f$ in $S \coprod_{U} T$ and dually for $e, f \in \mathcal{E}(T)$.

Proof. By Theorem 9.8, the inclusion of $S$ into $S \coprod_{U} T$ is the identity on vertices whence it gives an idempotent-separating inverse semigroup homomorphism onto its image (which is an inductive ordered subgroupoid of $\left.S \coprod_{U} T\right)$. But, by Hall's Theorem [4], the inclusion of $S$ into $S *_{U} T$ is an embedding. Since this inclusion factors through $\varphi$, it follows that $S$ embeds into $S \coprod_{U} T$. The result for $T$ is dual.

As to the second statement suppose $g \in \mathcal{E}(T) \backslash \mathcal{E}(U)$ and ef $\leq g \leq e, f$. Then there exist $u, u^{\prime} \in \mathcal{E}(U)$ with $g \leq u \leq e$ and $g \leq u^{\prime} \leq f$ whence ef $\leq g \leq u u^{\prime} \leq e f$. It follows that $g=u u^{\prime}$, a contradiction. We conclude ef is the meet of $e, f$ in $S \coprod_{U} T$; a dual argument holds if $e, f \in \mathcal{E}(T)$.

By [7, Theorem 4.1.9], there is an inductive groupoid $I$ so that $S \coprod_{U} T$ sits inside $I$ as an ordered subgroupoid and the inclusion preserves whatever meets exist in $S \coprod_{U} T$. It follows from the above lemma that $S$ and $T$ live as inverse subsemigroups of $I$ whence there is a morphism $\tau: S{ }{ }_{U} T \rightarrow I$. Note that $\tau \varphi$ is the identity on $S$ and $T$ and hence, by the universal property, on $S \coprod_{U} T$.

Lemma 9.14. Let $(S, U, T)$ be a tame amalgam of inductive groupoids. Then $\left.\varphi\right|_{V\left(S \amalg_{U} T\right)}$ is an order embedding. 
Proof. Suppose $v, w \in V\left(S \coprod_{U} T\right)$ with $\varphi(v) \leq \varphi(w)$. Then

$$
v=\tau(\varphi(v)) \leq \tau(\varphi(w))=w
$$

and the result follows.

Observe that $\varphi\left(S \coprod_{U} T\right)$ is an ordered subgroupoid of $S *_{U} T$ since, by Lemma 9.14, $\varphi$ is an order embedding on the vertices. The following technical lemma will be important in proving our result:

Lemma 9.15. Let $(S, U, T)$ be a tame amalgam of inductive groupoids. Suppose $e \in V\left(S \coprod_{U} T\right), y \in S *_{U} T$ and $e \leq \mathbf{d}(y)$. Then ey $\in \varphi\left(S \coprod_{U} T\right)$.

Proof. We induct on the length of a factorization of $y$ as a product of elements of $S \cup T$. If $y$ has length 1 , then $y \in S \cup T$. Since $\varphi$ is an order embedding, $e \leq y$ in $S \coprod_{U} T$ whence $(e \mid y)$ exists in $S \coprod_{U} T$. But then $\varphi(e \mid y) \leq y$ and $\mathbf{d}(\varphi(e \mid y))=e$ so $e y=\varphi(e \mid y)$. Suppose, without loss of generality, $y=s z$ with $s \in S$ and where $z$ has factorization of length $n-1$ as a product of elements of $S \cup T$. Then $e \leq \mathbf{d}(y) \leq \mathbf{d}(s)$, so, by the above case, $e s=\varphi(w)$. Note that $e y=(e s) z$ and $\mathbf{d}(e y)=e=\mathbf{d}(e s)$. Thus

$$
\mathbf{r}(e s)=s^{-1} e s=s^{-1} \mathbf{d}(e s z) s=\left(s^{-1} e s\right) z z^{-1}\left(s^{-1} e s\right) \leq \mathbf{d}(z) .
$$

Since $\mathbf{r}(e s)=\mathbf{r}(\varphi(w))=\mathbf{r}(w) \in V\left(S \coprod_{U} T\right), \mathbf{r}(w) z=\varphi(v)$ for some $v \in$ $S \coprod_{U} T$ by induction, and, necessarily, $\mathbf{d}(v)=\mathbf{r}(e s)=\mathbf{r}(w)$. But then

$$
e y=e s z=e s \mathbf{r}(e s) z=(e s)(\mathbf{r}(w) z)=\varphi(w) \varphi(v)=\varphi(w v)
$$

and the result follows.

Theorem 9.16. Let $(S, U, T)$ be a tame amalgam of inductive groupoids. Then $S \coprod_{U} T$ embeds as a $\mathcal{D}$-saturated ordered subgroupoid of $S *_{U} T$. Thus if $e \in E(S) \cup E(T)$, then the maximal subgroup at $e$ is $\pi_{1}\left(\mathcal{G}_{v}, \Gamma_{v}\right)$. This applies, in particular, if $E(U)$ is an order ideal of $E(S)$ and $E(T)$, or $U$ is $\mathcal{D}$-saturated in $S$ and $T$.

Proof. Let $K$ be the $\mathcal{D}$-saturated subgroupoid of $S *_{U} T$ consisting of the $\mathcal{D}$-classes of elements of $S \cup T$. Notice this is an ordered subgroupoid of $S *_{U} T$ and that $\varphi\left(S \coprod_{U} T\right) \subseteq K$. We show that $\varphi: S \coprod_{U} T \rightarrow K$ is onto; it will then follow that $\tau$ takes $K$ to $S \coprod_{U} T$, and that $\left.\varphi \tau\right|_{K}$ is the identity thereby proving the theorem.

First observe that to prove $\varphi$ maps onto $K$, it suffices to show that it is a fibration; indeed, any edge or vertex of $K$ lies on a path starting at an element of $\mathcal{E}(S) \cup \mathcal{E}(T)$ and this path can be lifted under a fibration. Suppose then that $e \in \mathcal{E}(S) \cup \mathcal{E}(T)$ and $x \in K$ with $\mathbf{d}(x)=e$; then $e \leq \mathbf{d}(x)$, so Lemma 9.15 shows that $x=e x \in \varphi\left(S \coprod_{U} T\right)$.

Once again, this theorem completely describes the structure of the ordered subgroupoid of $S *_{U} T$ consisting of the $\mathcal{D}$-classes of idempotents of $S$ or $T$. 
In the case that $U$ is $\mathcal{D}$-saturated in $S$ and $T$ and $e \in \mathcal{E}(U)$, then the maximal subgroup at $e$ in $S$ and in $T$ are the same. The following corollary is then immediate from the remarks after Proposition 9.5:

Corollary 9.17. Let $(S, U, T)$ be an amalgam of inverse semigroups such that $U$ is $\mathcal{D}$-saturated in $S$ and $T$. Then, for $e \in \mathcal{E}(S)$ (respectively, $\mathcal{E}(T)$ ), the maximal subgroups at e in $S *_{U} T$ is the same as in $S$ (respectively, $T$ ).

The above situation occurs, for instance, if $S$ and $T$ have a common ideal $U$. Another possible application is when $S$ and $T$ have a common $\mathcal{D}$-class $U$ which is a group (for instance, the minimal ideal and the group of units are always $\mathcal{D}$-classes which are subgroups).

We end with a discussion of some constructions which are similar in spirit to amalgams and to which our techniques apply.

A map of inverse semigroups $\varphi: S \rightarrow T$ is said to be a prehomomorphism if $\varphi\left(s^{-1}\right)=\varphi(s)^{-1}$ and $\varphi(s t) \leq \varphi(s) \varphi(t)$. It is shown [7] that prehomomorphisms correspond to general ordered groupoid morphisms of inductive groupoids (as opposed to those preserving the meet). If $(S, U, T)$ is an inverse semigroup amalgam, then we define the pre-amalgamated product of $S$ and $T$ over $U$, denoted $S *_{U}^{\prime} T$, to be the pushout [9] of $S$ and $T$ over $U$ in the category of inverse semigroups and prehomomorphisms. It follows by considering the map from the pre-amalgamated product to the amalgamated product that $S$ and $T$ map injectively into the pre-amalgamated product with intersection $U$.

As a simple example, if $S$ and $T$ are inverse semigroups with 0 and $U=0$, then $S *_{U}^{\prime} T$ is the 0-direct union of $S$ and $T$, that is, the semigroup $S \cup T$ where all products of elements of $S$ with elements of $T$ are 0 .

Theorem 9.18. Suppose $(S, U, T)$ is an amalgam of inverse semigroups such that $\mathcal{E}(U)$ is an order ideal of $\mathcal{E}(S)$ and $\mathcal{E}(T)$, and $\mathcal{E}(S) \cup \mathcal{E}(T)$ is a semilattice. Then $S *_{U}^{\prime} T=S \coprod_{U} T$, the ordered groupoid amalgamated product of $S$ and $T$ over $U$, and the maximal subgroups can be described as per Theorem 9.8. Furthermore, the natural maps of $S$ and $T$ into $S *_{U}^{\prime} T$ are homomorphisms.

Proof. First observe that the hypotheses of Theorem 9.8 hold since $(S, U, T)$ is tame by Proposition 9.10. Our assumptions imply that $V\left(S \coprod_{U} T\right)$ is a semilattice so $S \coprod_{U} T$ is an inductive groupoid. The desired universal property is then automatic. The last statement is clear from the construction of $S \coprod_{U} T$.

Note that in the context of the above theorem, Theorem 9.16 implies that $S *_{U}^{\prime} T$ lives in $S *_{U} T$ as a $\mathcal{D}$-saturated ordered subgroupoid; but the inclusion does not in general preserve meets (think about the inclusion of $S *_{0}^{\prime} T$ into $S *_{0} T$ for inverse semigroups $S$ and $T$ with zeroes). Proposition 9.3 allows us to find examples of when the theorem applies. One example is the case 
where $S \cap T$ is a common ideal $U$ which is a monoid with identity $e$. The case of the 0-direct union is such. It is easy to show that in this case $S *_{U} T$ has the following structure: $S *_{U} T=S \cup T$ with the product in $S$ and $T$ as usual, and with the product $s t=(s e)(e t)$ for $s \in S, t \in T$. In this case, of course, the structure of the maximal subgroups is transparent: They are just the maximal subgroups of $S$ and $T$.

A more general example is if $S$ and $T$ have ideals $U_{S}, U_{T}$ (respectively) which are monoids with a common full inverse submonoid $U$ (so $E\left(U_{S}\right)=$ $\left.E(U)=E\left(U_{T}\right)\right)$. In this case, the theorem really does tell us something about the structure of the maximal subgroups. The simplest example is when $S$ and $T$ have minimal ideals and $U$ is a common subgroup of the minimal ideals.

We mention that Yamamura [25] defines a notion of a graph of full inverse monoids and the fundamental inverse monoid of such. Full amalgams and full HNN-extensions are special cases of this construction. In the paper, he obtains a graph of groups decomposition of the maximal subgroups of such a fundamental inverse monoid similar to that for full amalgams. We leave it as an exercise for the reader to obtain these results by realizing the fundamental inverse monoid as the fundamental ordered groupoid of an ordered 2-complex with an obvious graph of 2-complexes decomposition and then using Theorem 9.6.

We conjecture that actions of inverse semigroups on ordered forests, as per [25], can be completely classified using ordered 2-complexes and the usual translation between Bass-Serre theory and graphs and trees of 2complexes. In particular, we guess the solution will obtain the structure of such as the fundamental ordered groupoid of an ordered graph of groups.

\section{Appendix.}

Since this paper was written and submitted, a survey [23] of the results in this paper has been submitted to the proceedings of a school held in Coimbra, Portugal during May and June of 2001. This survey offers many illustrative examples (with figures). Also a slight improvement has been made in the definition of the standard 2-complex so that cells whose boundaries are labeled by Dyck words are removed. In addition to streamlining the construction (and making sure that it agrees exactly with the group theoretic construction in the case of a group), this allows us to formulate a slightly stronger version of Theorem 8.10.

\section{References}

[1] P. Bennett, On the structure of inverse semigroup amalgams, Internat. J. Algebra. Comput., 7 (1998), 577-604, MR 98g:20095, Zbl 0892.20033. 
[2] C. Ehresmann, Oeuvres Complète et Commentées, in 'Supplements to Cahiers de Topologie et Géométrie Différentielle' (ed. A.C. Ehresmann), Amiens, 1980-83, MR 86i:01059, Zbl 0561.01027.

[3] S. Haataja, S.W. Margolis and J. Meakin, Bass-Serre theory for groupoids and the structure of full regular semigroup amalgams, J. Algebra, 183 (1996), 38-54, MR 97e:20076, Zbl 0858.20055.

[4] T.E. Hall, Free products with amalgamation of inverse semigroups, J. Algebra, 34 (1975), 375-385, MR 52 \#3401, Zbl 0326.20054.

[5] _ Amalgamation of inverse and regular semigroups, Tran. Amer. Math. Soc., 246 (1978), 395-406, MR 80d:20053, Zbl 0401.20053.

[6] K. Krohn, J. Rhodes and B. Tilson, Lectures on the algebraic theory of finite semigroups and finite-state machines, Chapters 1, 5-9 (Chapter 6 with M.A. Arbib) in 'The Algebraic Theory of Machines, Languages, and Semigroups', (M.A. Arbib, ed.), Academic Press, New York, 1968.

[7] M.V. Lawson, Inverse Semigroups: The Theory of Partial Symmetries, World Scientific, Singapore, 1998, MR 2000g:20123.

[8] R.C. Lyndon and P.E. Schupp, Combinatorial Group Theory, Springer-Verlag, New York, 1977, MR 58 \#28182, Zbl 0368.20023.

[9] S. MacLane, Categories for the Working Mathematician, Springer-Verlag, New York, 1971, MR 50 \#7275, Zbl 0705.18001.

[10] D.B. McAlister, Groups, semilattices and inverse semigroups II, Trans. Amer. Math. Soc., 196 (1974), 231-251, MR 50 \#10128, Zbl 0297.20072.

[11] K.S.S. Nambooripad, Structure of regular semigroups I, Mem. Amer. Math Soc., 22(224) (1979), MR 81i:20086, Zbl 0457.20051.

[12] K.S.S. Nambooripad and F.J. Pastijn, Amalgamation of regular semigroups, Houston J. of Math., 15 (1989), 249-254, MR 91g:20093, Zbl 0697.20048.

[13] E.T. Ordman, On subgroups of amalgamated free products, Proc. Cambridge Phil. Soc., 69 (1971), 13-23, MR 43 \#2102, Zbl 0203.32303.

[14] _ Amalgamated free products of groupoids, Ph.D. Thesis, Princeton University.

[15] A.L.T. Paterson, Groupoids, Inverse Semigroups, and Their Operator Algebras, Birkhäuser, Boston, 1999, MR 2001a:22003, Zbl 0913.22001.

[16] G.B. Preston, Inverse semi-groups, J. London Math. Soc., 29 (1954), 396-403, MR 16,215c, Zbl 0056.01903.

[17] N. Ruskuc, Presentations for subgroups of monoids, J. Algebra, 220 (1999), 365-380, MR 2000h:20105, Zbl 0943.20057.

[18] B.M. Schein, On the theory of inverse semigroups and generalized groups, American Math. Translations (2), 113 (1979), 89-122, Zbl 0404.20055.

[19] J.-P. Serre, Trees, Springer-Verlag, Heidelberg, 1980, MR 82c:20083, Zbl 0548.20018.

[20] J. Stallings, Topology of finite graphs, Invent. Math., 71 (1983), 551-565, MR 85m:05037a, Zbl 0521.20013.

[21] B. Steinberg, Factorization theorems for morphisms of ordered groupoids and inverse semigroups, Proc. Edinburgh Math. Soc., 44 (2001), 549-569, MR 2002m:20098, Zbl 0990.20043.

[22] _ A note on amalgams of inverse semigroups, J. Austral. Math. Soc., 70 (2001), 71-75, MR 2001j:20098, Zbl 0982.20053. 
[23] _ A survey of a topological approach to inverse semigroups, in 'Semigroup, Algorithms and Languages' (G. M. S. Gomes, J.-E. Pin, and P. V. Silva, eds.), World Scientific, to appear.

[24] J.B. Stephen, Presentations of inverse monoids, J. Pure Appl. Algebra ,63 (1990), 81-112, MR 91g:20083, Zbl 0691.20044.

[25] A. Yamamura, A class of inverse monoids acting on ordered forests, preprint, 2000.

Received June 6, 2001 and revised December 14, 2001. The author was supported in part by NSF-NATO postdoctoral fellowship DGE-9972697 and by FCT through Centro de Matemática da Universidade do Porto.

Departmento de Matemática Pura

FACULDADE DE CiÊnCIAS

DA Universidade do Porto

4099-002 PORTO

Portugal

E-mail address: bsteinbg@agc0.fc.up.pt 